Document downloaded from:

http://hdl.handle.net/10251/71238

This paper must be cited as:

ANGELA ALBANESE; Bonet Solves, JA.; WERNER RICKER (2015). On the continuous Cesàro operator in certain function spaces. Positivity. 19:659-679. doi:10.1007/s11117-0140321-5.

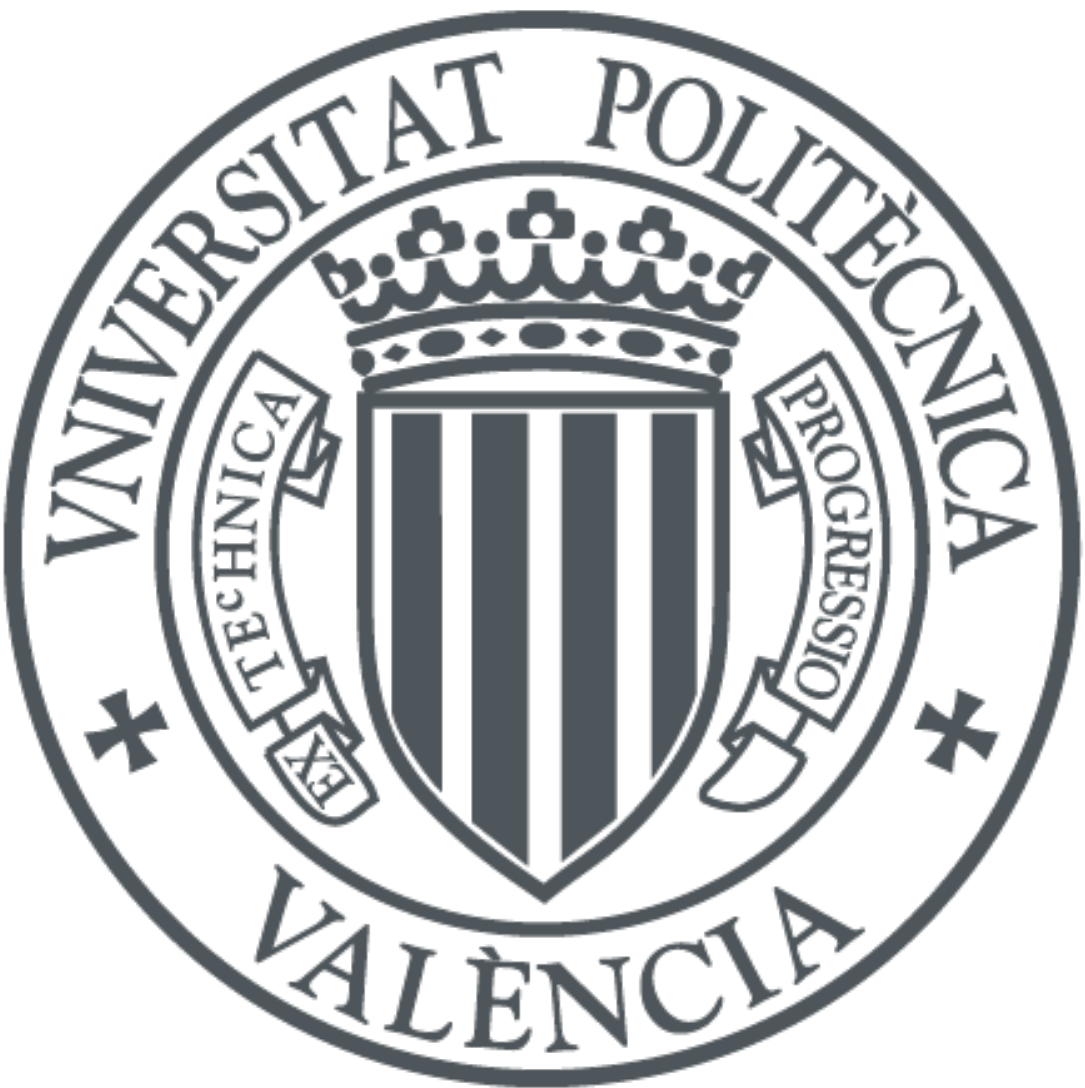

The final publication is available at

http://dx.doi.org/10.1007/s11117-014-0321-5

Copyright Springer Verlag (Germany)

Additional Information

"The final publication is available at Springer via http://dx.doi.org/10.1007/s11117-014-03215" 


\title{
ON THE CONTINUOUS CESĀRO OPERATOR IN CERTAIN FUNCTION SPACES
}

\author{
ANGELA A. ALBANESE, JOSÉ BONET AND WERNER J. RICKER
}

\begin{abstract}
Various properties of the (continuous) Cesàro operator C, acting on Banach and Fréchet spaces of continuous functions and $L^{p}$-spaces, are investigated. For instance, the spectrum and point spectrum of $C$ are completely determined and a study of certain dynamics of $C$ is undertaken (eg. hyperand supercyclicity, chaotic behaviour). In addition, the mean (and uniform mean) ergodic nature of $C$ acting in the various spaces is identified.
\end{abstract}

\section{INTRODUCTION}

Let $f$ be a $\mathbb{C}$-valued, locally integrable function defined on $\mathbb{R}^{+}:=[0, \infty)$. Then the Cesàro average $\mathrm{C} f$ of $f$ is the function defined by

$$
\mathrm{C} f(x):=\frac{1}{x} \int_{0}^{x} f(t) d t, \quad x \in(0, \infty) .
$$

The linear map $f \mapsto \mathrm{C} f$ is called the continuous Cesàro operator (as distinct from the discrete Cesàro operator which forms the sequence of averages of vectors coming from various Banach sequence spaces) and has been intensively investigated in such Banach spaces as $L^{p}([0,1])$ and $L^{p}\left(\mathbb{R}^{+}\right)$, for $1<p<\infty$. The boundedness of $C$ on these spaces is due to G.H. Hardy, [19, p.240], who showed that the operator norm $\|\mathrm{C}\|_{o p}=q$ in both $L^{p}([0,1])$ and $L^{p}\left(\mathbb{R}^{+}\right)$, where $\frac{1}{p}+\frac{1}{q}=1$. The spectra and point spectra of $C$ are also known; see [11], [12], [21], [22], for example, and the references therein. Two further Banach spaces on which $\mathrm{C}$ is naturally defined are the spaces of continuous functions $C([0,1])$ and $C_{l}([0, \infty])$, both equipped with the sup-norm; here $C_{l}([0, \infty])$ is the space of all $\mathbb{C}$-valued, continuous functions $f$ on $\mathbb{R}^{+}$for which $f(\infty):=\lim _{x \rightarrow \text { infty }} f(x)$ exists in $\mathbb{C}$. In both spaces $\|\mathrm{C}\|_{o p}=1$. The spectrum and point spectrum of $\mathrm{C}$ acting in these spaces are completely determined in Propositions 2.1 and 2.2.

The dynamics of $C$ have also been investigated in recent years. Recall that a bounded linear operator $T$, defined on a separable Banach space $X$ (or, more generally, a locally convex Hausdorff space $X$, briefly lcHs), is said to be hypercyclic if there exists $x \in X$ such that its orbit $\left\{T^{n} x: n \in \mathbb{N}_{0}\right\}$ is dense in $X$. Also, $T$ is called supercyclic if, for some $x \in X$, the projective orbit $\left\{\lambda T^{n} x: \lambda \in \mathbb{C}, n \in \mathbb{N}_{0}\right\}$ is dense in $X$. Finally, $T$ is said to be chaotic if it is hypercyclic and the set of periodic points $\left\{u \in X: \exists n \in \mathbb{N}\right.$ with $\left.T^{n} u=u\right\}$ is dense in $X$. As general references we refer to [8], [18], for example. It is known that C acting on $L^{p}([0,1])$,

Key words and phrases. Cesàro operator, continuous function spaces, $L^{p}$-spaces, (uniformly) mean ergodic operator, hypercyclic operator, supercyclic operator.

Mathematics Subject Classification 2010: Primary: 47A10, 47A16, 47A35; Secondary: 46A04, 47B34, 47B38. 
$1<p<\infty$, is hypercyclic and chaotic, [23], and that it is not (weakly) supercyclic in $L^{2}\left(\mathbb{R}^{+}\right)$, [17]. On the other hand, $C$ is not supercyclic (hence, not hypercyclic) on $C([0,1])$, [23]. We continue an investigation of such properties. For instance, in Proposition 2.7 it is shown that $\mathrm{C}$ is not supercyclic on $C_{l}([0, \infty])$.

There are also two natural types of Fréchet spaces in which the Cesàro operator $\mathrm{C}$ acts continuously. One is the Fréchet space $C\left(\mathbb{R}^{+}\right)$consisting of all $\mathbb{C}$-valued, continuous functions on $\mathbb{R}^{+}$endowed with the topology of uniform convergence on the compact subsets of $\mathbb{R}^{+}$. In this space the spectrum of $C$ is completely determined and it is shown that $\mathrm{C}$ is not supercyclic; see Theorem 3.1. The other class of Fréchet spaces consists of the reflexive spaces $L_{l o c}^{p}\left(\mathbb{R}^{+}\right), 1<p<\infty$, consisting of all $\mathbb{C}$-valued, measurable functions on $\mathbb{R}^{+}$which are $p$-th power integrable on each set $[0, j]$, for $j \in \mathbb{N}$. In these spaces the spectrum of $C$ is also determined and it is shown that $\mathrm{C}$ is chaotic (cf. Theorem 4.2).

The main point of departure of this paper is actually to investigate (various) mean ergodic properties of $\mathrm{C}$. Let $X$ be a lcHs and $\Gamma_{X}$ be a system of continuous seminorms determining the topology of $X$. The strong operator topology $\tau_{s}$ in the space $\mathcal{L}(X)$ of all continuous linear operators from $X$ into itself (from $X$ into another lcHs $Y$ we write $\mathcal{L}(X, Y))$ is determined by the family of seminorms $q_{x}(S):=q(S x)$, for $S \in \mathcal{L}(X)$, for each $x \in X$ and $q \in \Gamma_{X}$, in which case we write $\mathcal{L}_{s}(X)$. Denote by $\mathcal{B}(X)$ the collection of all bounded subsets of $X$. The topology $\tau_{b}$ of uniform convergence on bounded sets is defined in $\mathcal{L}(X)$ via the seminorms $q_{B}(S):=\sup _{x \in B} q(S x)$, for $S \in \mathcal{L}(X)$, for each $B \in \mathcal{B}(X)$ and $q \in \Gamma_{X}$; in this case we write $\mathcal{L}_{b}(X)$. For $X$ a Banach space, $\tau_{b}$ is the operator norm topology in $\mathcal{L}(X)$. If $\Gamma_{X}$ is countable and $X$ is complete, then $X$ is called a Fréchet space. The identity operator on a lcHs $X$ is denoted by $I$. Finally, the dual operator of $T \in \mathcal{L}(X)$ is denoted by $T^{\prime}: X^{\prime} \rightarrow X^{\prime}$, where $X^{\prime}=\mathcal{L}(X, \mathbb{C})$ is the topological dual space of $X$. As a general reference for lcHs' see [25].

The relevant classes of operators are as follows. We say that $T \in \mathcal{L}(X)$, with $X$ a lcHs, is power bounded if $\left\{T^{n}\right\}_{n=1}^{\infty}$ is an equicontinuous subset of $\mathcal{L}(X)$. For $X$ a Banach space, this means precisely that $\sup _{n \in \mathbb{N}}\left\|T^{n}\right\|_{o p}<\infty$. Given $T \in \mathcal{L}(X)$, we can consider its sequence of averages

$$
T_{[n]}:=\frac{1}{n} \sum_{m=1}^{n} T^{m}, \quad n \in \mathbb{N}
$$

called the Cesàro means of $T$. Then $T$ is called mean ergodic (resp., uniformly mean ergodic) if $\left\{T_{[n]}\right\}_{n=1}^{\infty}$ is a convergent sequence in $\mathcal{L}_{s}(X)$ (resp., in $\mathcal{L}_{b}(X)$ ). Since $\frac{T^{n}}{n}=T_{[n]}-\frac{n-1}{n} T_{[n-1]}$, for $n \geq 2$, it is clear that $\tau_{s}-\lim _{n \rightarrow \infty} \frac{T^{n}}{n}=0$ whenever $T$ is mean ergodic. Hence, a mean ergodic operator acting in a Banach space always has its spectrum lying in $\{z \in \mathbb{C}:|z| \leq 1\}$, [14, p.709, Lemma 1]. The study of mean ergodic operators, initiated by J. von Neumann, N. Dunford, F. Riesz and others, began in the 1930's and has continued ever since; see [20], [30, Ch. VIII] and the references therein. In Theorem 2.3 it is shown that C acting on the Banach space $C([0,1])$ is power bounded and mean ergodic but, fails to be uniformly mean ergodic, whereas in the Banach space $C_{l}([0, \infty])$ the Cesàro operator $\mathrm{C}$ is power bounded but, not even mean ergodic (cf. Theorem 2.6). Concerning the above mentioned classes of Fréchet spaces in which $C$ acts continuously, it is shown in Theorem 3.1 that $\mathrm{C}$ is both power bounded and mean 
ergodic in $C\left(\mathbb{R}^{+}\right)$but, not uniformly mean ergodic. Finally, in the Fréchet spaces $L_{l o c}^{p}\left(\mathbb{R}^{+}\right), 1<p<\infty$, it turns out that $C$ is neither power bounded nor mean ergodic (cf. Theorem 4.2). For recent results on mean ergodic operators in lcHs' we refer to [4], [5], [6], [7], [28], [29], for example, and the references therein.

For a Fréchet space $X$ and $T \in \mathcal{L}(X)$, the resolvent set $\rho(T)$ of $T$ consists of all $\lambda \in \mathbb{C}$ such that $R(\lambda, T):=(\lambda I-T)^{-1}$ exists in $\mathcal{L}(X)$. Then $\sigma(T):=\mathbb{C} \backslash \rho(T)$ is called the spectrum of $T$. The point spectrum $\sigma_{p t}(T)$ consists of all $\lambda \in \mathbb{C}$ such that $(\lambda I-T)$ is not injective. If we need to stress the space $X$, then we also write $\sigma(T ; X), \sigma_{p t}(T ; X)$ and $\rho(T ; X)$. Unlike for Banach spaces, it may happen that $\rho(T)=\emptyset$. For example, let $\omega=\mathbb{C}^{\mathbb{N}}$ be the Fréchet space equipped with the lctopology determined via the seminorms $\left\{q_{n}\right\}_{n=1}^{\infty}$, where $q_{n}(x):=\max _{1 \leq j \leq n}\left|x_{j}\right|$, for $x=\left(x_{j}\right)_{j=1}^{\infty} \in \omega$. Then the unit left shift operator $T: x \mapsto\left(x_{2}, x_{3}, x_{4}, \ldots\right)$, for $x \in \omega$, belongs to $\mathcal{L}(\omega)$ and, for every $\lambda \in \mathbb{C}$, the element $\left(1, \lambda, \lambda^{2}, \lambda^{3}, \ldots\right) \in \omega$ is an eigenvector corresponding to $\lambda$. Or, let $A=\left\{\alpha_{n}: n \in \mathbb{N}\right\}$ be any countable subset of $\mathbb{C}$ and define $S \in \mathcal{L}(\omega)$ by $S: x \mapsto\left(\alpha_{1} x_{1}, \alpha_{2} x_{2}, \alpha_{3} x_{3}, \ldots\right)$, for $x \in \omega$. Then $\sigma(S)=\sigma_{p t}(S)=A$ and hence, $\sigma(S)$ need not even be a closed subset of $\mathbb{C}$.

For ease of reading, some technical (but useful) results which are needed in relation to the spectrum and mean ergodicity of continuous linear operators acting in the class of Fréchet spaces called quojections (to which $C\left(\mathbb{R}^{+}\right)$and $L_{l o c}^{p}\left(\mathbb{R}^{+}\right.$), $1<p<\infty$, belong) have been formulated in an Appendix at the end of the paper.

\section{The Cesàro operator on Banach spaces of Continuous functions}

We consider here the continuous Cesàro operator $C$ given in (1.1) when acting on the Banach spaces $C([0,1])$ and $C_{l}([0, \infty])$.

In order to make the definition of the operator $\mathrm{C}$ consistent, we set $\mathrm{C} f(0):=$ $\lim _{x \rightarrow 0^{+}} \mathrm{C} f(x)=f(0)$ for every $f \in C([0,1])$ or $f \in C_{l}([0, \infty])$. It is routine to check if $f \in C_{l}([0, \infty])$, then also $\lim _{x \rightarrow \infty} \mathrm{C} f(x)$ exists and equals $f(\infty):=$ $\lim _{x \rightarrow \infty} f(x)$, i.e., $C f(\infty)=f(\infty)$. Then the linear maps $\mathrm{C}: C([0,1]) \rightarrow C([0,1])$ and $\mathrm{C}: C_{l}([0, \infty]) \rightarrow C_{l}([0, \infty])$ are well defined with $\|\mathrm{C}\|_{o p}=1$ and satisfy $\mathrm{C} 1=$ $\mathbf{1}$, where $\mathbf{1}$ is the constant function equal to 1 . Moreover, the null space $\operatorname{Ker}(I-$ $\mathrm{C})=\operatorname{span}\{\mathbf{1}\}$. Indeed, every function $f$ satisfying $C f=f$ must be continuously differentiable on $(0,1)$ or $(0, \infty)$ via $(1.1)$; apply the quotient rule to deduce from (1.1) and $(\mathrm{C} f)^{\prime}=f^{\prime}$ that $f^{\prime} \equiv 0$.

We begin by identifying the spectrum and point spectrum of the Cesàro operator $\mathrm{C}$ on the Banach spaces $C([0,1])$ and $C_{l}([0, \infty])$.

Proposition 2.1. The Cesàro operator $\mathrm{C}: C([0,1]) \rightarrow C([0,1])$ satisfies

$$
\sigma(\mathrm{C} ; C([0,1]))=\left\{\lambda \in \mathbb{C}:\left|\lambda-\frac{1}{2}\right| \leq \frac{1}{2}\right\}
$$

and

$$
\sigma_{p t}(\mathrm{C} ; C([0,1]))=\left\{\lambda \in \mathbb{C}:\left|\lambda-\frac{1}{2}\right| \leq \frac{1}{2}\right\} \backslash\{0\} .
$$

Proof. It is routine to check that $\mathrm{C}$ is injective on $C([0,1])$. Also, $\mathrm{C}$ is not surjective (the range of $\mathrm{C}$ contains only continuously differentiable functions on $(0,1])$. Hence, $0 \in \sigma(\mathrm{C} ; C([0,1])) \backslash \sigma_{p t}(\mathrm{C} ; C([0,1]))$. If $\lambda \in \mathbb{C} \backslash\{0\}$ satisfies $\left|\lambda-\frac{1}{2}\right| \leq \frac{1}{2}$, then the function $g_{\lambda}(x):=x^{\frac{1}{\lambda}-1}$, for $x \in[0,1]$, belongs to $C([0,1])$ and $C g_{\lambda}=\lambda g_{\lambda}$, 
i.e., $\lambda \in \sigma_{p t}(\mathrm{C} ; C([0,1]))$. If $\lambda \in \mathbb{C}$ satisfies $\left|\lambda-\frac{1}{2}\right|>\frac{1}{2}$ (equivalently, $\operatorname{Re}\left(\frac{1}{\lambda}\right)<1$ ), then for $\xi:=\frac{1}{\lambda}$ the linear map

$$
P_{\xi} f(x):=\int_{0}^{1} s^{-\xi} f(x s) d s, \quad x \in[0,1]
$$

is a bounded operator on $L^{\infty}([0,1])$ with the property that $\xi I+\xi^{2} P_{\xi}$ is the inverse of $(\lambda I-\mathrm{C})$ on $L^{\infty}([0,1])$; see [11] and the comments on p.29 of [21]. By the dominated convergence theorem applied to calculating $\lim _{n \rightarrow \infty} P_{\xi} f\left(x_{n}\right)$ whenever $f \in C([0,1])$ and $x_{n} \rightarrow x$ in $[0,1]$ for $n \rightarrow \infty$, it follows that $P_{\xi} f \in C([0,1])$ whenever $f \in C([0,1])$, i.e., $\xi I+\xi^{2} P_{\xi}$ restricted to the closed invariant subspace $C([0,1])$ of $L^{\infty}([0,1])$ is the inverse of $(\lambda I-\mathrm{C})$ restricted from $L^{\infty}([0,1])$ to $C([0,1])$. This implies that $\lambda \notin \sigma(\mathrm{C} ; C([0,1]))$. So, the proof is complete.

Proposition 2.2. The Cesàro operator $\mathrm{C}: C_{l}([0, \infty]) \rightarrow C_{l}([0, \infty])$ satisfies

$$
\sigma\left(\mathrm{C} ; C_{l}([0, \infty])\right)=\left\{\lambda \in \mathbb{C}:\left|\lambda-\frac{1}{2}\right|=\frac{1}{2}\right\}
$$

and

$$
\sigma_{p t}\left(\mathrm{C} ; C_{l}([0, \infty])\right)=\{1\} .
$$

Proof. Since $\mathbf{C} \mathbf{1}=\mathbf{1}$, we have $1 \in \sigma_{p t}\left(\mathrm{C} ; C_{l}([0, \infty])\right)$. The same argument given in the proof of Proposition 2.1 yields that $0 \in \sigma\left(\mathrm{C} ; C_{l}([0, \infty])\right) \backslash \sigma_{p t}\left(\mathrm{C} ; C_{l}([0, \infty])\right)$. If $f \in C_{l}([0, \infty])$ satisfies $C f=\lambda f$ for some $\lambda \neq 0$, then $f$ is continuously differentiable in $(0, \infty)$ and is a solution of the 1-st order Euler differential equation $\lambda x y^{\prime}(x)+(\lambda-1) y(x)=0$. But, every solution of this ODE has the form $f(x)=\beta x^{\frac{1}{\lambda}-1}, x \in \mathbb{R}^{+}$, for some $\beta \in \mathbb{C}$. Since $x^{\alpha} \notin C_{l}([0, \infty])$ unless $\alpha=0$, we conclude that necessarily $\lambda=1$. Thus $\sigma_{p t}\left(\mathrm{C} ; C_{l}([0, \infty])\right)=\{1\}$.

Now we prove that

$$
\sigma\left(\mathrm{C} ; C_{l}([0, \infty])\right)=\left\{\lambda \in \mathbb{C}:\left|\lambda-\frac{1}{2}\right|=\frac{1}{2}\right\}=\left\{\lambda \in \mathbb{C}: \operatorname{Re}\left(\frac{1}{\lambda}\right)=1\right\} .
$$

For this we recall that D. Boyd proved the following results, [11, Theorem 1]:

(1) If $\operatorname{Re}(\xi)<1$, then $P_{\xi} f(x):=\int_{0}^{1} s^{-\xi} f(x s) d s$, for $x \in \mathbb{R}^{+}$, defines a continuous linear operator on $L^{\infty}\left(\mathbb{R}^{+}\right)$. Moreover, if $\lambda \neq 0$ and $\operatorname{Re}\left(\frac{1}{\lambda}\right)<$ 1 , then

$$
(\lambda I-\mathrm{C})^{-1}=\left(\lambda^{-1} I+\lambda^{-2} P_{1 / \lambda}\right), \quad \text { in } \mathcal{L}\left(L^{\infty}\left(\mathbb{R}^{+}\right)\right) .
$$

(2) If $\operatorname{Re}(\xi)>1$, then $Q_{\xi} f(x):=\int_{1}^{\infty} s^{-\xi} f(x s) d s$, for $x \in \mathbb{R}^{+}$, defines a continuous linear operator on $L^{\infty}\left(\mathbb{R}^{+}\right)$. Moreover, if $\lambda \neq 0$ and $\operatorname{Re}\left(\frac{1}{\lambda}\right)>$ 1 , then

$$
(\lambda I-C)^{-1}=\left(\lambda^{-1} I+\lambda^{-2} Q_{1 / \lambda}\right), \text { in } \mathcal{L}\left(L^{\infty}\left(\mathbb{R}^{+}\right)\right) .
$$

To show that

$$
\sigma\left(\mathrm{C} ; C_{l}([0, \infty])\right) \subseteq\left\{\lambda \in \mathbb{C}: \operatorname{Re}\left(\frac{1}{\lambda}\right)=1\right\},
$$

we first observe, via the dominated convergence theorem (as applied in the proof of Proposition 2.1), that if $f$ is bounded and continuous on $\mathbb{R}^{+}$, then also $P_{\xi} f$, for $\operatorname{Re}(\xi)<1$, and $Q_{\xi} f$, for $\operatorname{Re}(\xi)>1$, are bounded and continuous functions on $\mathbb{R}^{+}$. So, the proof of $(2.1)$ will follow if we can show, for each $f \in C_{l}([0, \infty])$, 
that $P_{\xi} f \in C_{l}([0, \infty])$ whenever $\operatorname{Re}(\xi)<1$ and that $Q_{\xi} f \in C_{l}([0, \infty])$ whenever $\operatorname{Re}(\xi)>1$. To see this, fix $f \in C_{l}([0, \infty])$. Take first $\xi \in \mathbb{C}$ with $\operatorname{Re}(\xi)<1$. Fix a sequence $\left\{x_{n}\right\}_{n=1}^{\infty} \subseteq(0, \infty)$ such that $x_{n} \rightarrow \infty$. Then $f\left(x_{n} s\right) \rightarrow f(\infty)$, for every fixed $s \in(0,1)$ as $n \rightarrow \infty$, and $\left|s^{-\xi} f\left(x_{n} s\right)\right| \leq s^{-\operatorname{Re}(\xi)}\|f\|_{\infty}$ for all $s \in(0,1)$ and $n \in \mathbb{N}$ with $s^{-\operatorname{Re}(\xi)} \in L^{1}([0,1])$ as $\operatorname{Re}(\xi)<1$. Then the dominated convergence theorem implies that $P_{\xi} f\left(x_{n}\right)=\int_{0}^{!} 1 s^{-\xi} f\left(x_{n} s\right) d s \rightarrow \int_{0}^{1} s^{-\xi} f(\infty) d s$ as $n \rightarrow \infty$. Since the sequence $\left\{x_{n}\right\}_{n=1}^{\infty}$ is arbitrary, it follows that $\lim _{x \rightarrow \infty} P_{\xi} f(x)$ exists and is equal to $\frac{f(\infty)}{1-\xi}$. So, $P_{\xi} f \in C_{l}([0, \infty])$.

Now, let $\xi \in \mathbb{C}$ with $\operatorname{Re}(\xi)>1$. Fix again a sequence $\left\{x_{n}\right\}_{n=1}^{\infty} \subseteq(0, \infty)$ such that $x_{n} \rightarrow \infty$. Then $f\left(x_{n} s\right) \rightarrow f(\infty)$, for each fixed $s \in(1, \infty)$ as $n \rightarrow \infty$, and $\left|s^{-\xi} f\left(x_{n} s\right)\right| \leq s^{-\operatorname{Re}(\xi)}\|f\|_{\infty}$ for all $s \in(1, \infty)$ and $n \in \mathbb{N}$ with $s^{-\operatorname{Re}(\xi)} \in$ $L^{1}((1, \infty))$ as $\operatorname{Re}(\xi)>1$. Then the dominated convergence theorem implies that $Q_{\xi} f\left(x_{n}\right)=\int_{1}^{\infty} s^{-\xi} f\left(x_{n} s\right) d s \rightarrow \int_{1}^{\infty} s^{-\xi} f(\infty) d s$ as $n \rightarrow \infty$. Since the sequence $\left\{x_{n}\right\}_{n=1}^{\infty}$ is arbitrary, it follows that $\lim _{x \rightarrow \infty} Q_{\xi} f(x)$ exists and is equal to $\frac{f(\infty)}{\xi-1}$. So, $Q_{\xi} f \in C_{l}([0, \infty])$.

We will also require the fact that $\left\|P_{\xi}\right\|_{o p}=\frac{1}{1-\operatorname{Re}(\xi)}$ for each $\xi \in \mathbb{C}$ with $\operatorname{Re}(\xi)<$ 1. To verify this, fix $\xi \in \mathbb{C}$ with $\operatorname{Re}(\xi)<1$. If $f \in C_{l}([0, \infty])$ and $x \in \mathbb{R}^{+}$, then it follows from the definition of $P_{\xi} f$ that $\left|P_{\xi} f(x)\right| \leq \frac{1}{1-\operatorname{Re}(\xi)}\|f\|_{\infty}$. So, $\left\|P_{\xi}\right\|_{o p} \leq \frac{1}{1-\operatorname{Re}(\xi)}$. Since $P_{\xi} \mathbf{1}(x)=\frac{1}{1-\xi}$, for $x \in \mathbb{R}^{+}$, we are done if $\xi \in \mathbb{R}$ with $\xi<1$. For the remaining case, assume $\xi=\alpha+i \beta$, with $\beta \neq 0$ and $\alpha<1$. For given $\varepsilon>0$, define $g_{\varepsilon}(x):=x^{i \beta+\varepsilon}$ if $x \in(0,1], g_{\varepsilon}(0):=0$ and $g_{\varepsilon}(x):=1$ if $x \geq 1$. Then $g_{\varepsilon} \in C_{l}([0, \infty])$ and $\left\|g_{\varepsilon}\right\|_{\infty}=1$. Moreover,

$$
P_{\xi} g_{\varepsilon}(1)=\int_{0}^{1} s^{-\xi} g_{\varepsilon}(s) d s=\int_{0}^{1} s^{-\alpha+\varepsilon} d s=\frac{1}{1-\alpha+\varepsilon} .
$$

So, $\left\|P_{\xi} g_{\varepsilon}\right\|_{\infty} \geq \frac{1}{1-\alpha+\varepsilon}$. Hence, $\left\|P_{\xi}\right\|_{o p} \geq \sup _{\varepsilon>0} \frac{1}{1-\alpha+\varepsilon}=\frac{1}{1-\alpha}=\frac{1}{1-\operatorname{Re}(\xi)}$ and we can conclude, as stated, that $\left\|P_{\xi}\right\|_{o p}=\frac{1}{1-\operatorname{Re}(\xi)}$.

We complete the proof of $\sigma\left(\mathrm{C} ; C_{l}([0, \infty])\right)=\left\{\lambda \in \mathbb{C}: \operatorname{Re}\left(\frac{1}{\lambda}\right)=1\right\}$ by applying an argument of Boyd, [11, p.34]. Suppose there exists $\lambda_{0} \in \rho\left(\mathrm{C} ; C_{l}([0, \infty])\right)$ with $\lambda_{0} \in \mathbb{C} \backslash\{0,1\}$ and satisfying $\operatorname{Re}\left(\frac{1}{\lambda_{0}}\right)=1$. Select a sequence $\left\{\lambda_{n}\right\}_{n=1}^{\infty} \subseteq \mathbb{C}$ such that $\lambda_{n} \rightarrow \lambda_{0}$ for $n \rightarrow \infty$ and $\operatorname{Re}\left(\frac{1}{\lambda_{n}}\right)<1$ for all $n \in \mathbb{N}$. For each $n \in \mathbb{N}$, set $\xi_{n}:=\frac{1}{\lambda_{n}}$. Then

$$
\left\|\left(\lambda_{n} I-\mathrm{C}\right)^{-1}\right\|_{o p}=\left\|\xi_{n} I+\xi_{n}^{2} P_{\xi_{n}}\right\|_{o p} \geq\left|\xi_{n}\right|^{2} \frac{1}{1-\operatorname{Re}\left(\xi_{n}\right)}-\left|\xi_{n}\right|
$$

for every $n \in \mathbb{N}$. Since $\operatorname{Re}\left(\xi_{n}\right) \rightarrow 1$ as $n \rightarrow \infty$, it follows that $\|\left(\lambda_{n} I-\right.$ C) $)^{-1} \|_{o p} \rightarrow \infty$ for $n \rightarrow \infty$. This is a contradiction because the resolvent set $\rho\left(\mathrm{C} ; C_{l}([0, \infty])\right)$ is open in $\mathbb{C}$ and the resolvent map $\lambda \mapsto(\lambda I-\mathrm{C})^{-1}$ is continuous from $\rho\left(\mathrm{C} ; C_{l}([0, \infty])\right)$ into $\mathcal{L}_{b}\left(C_{l}([0, \infty])\right)$. So, no such $\lambda_{0}$ exists.

The mean ergodicity of $\mathrm{C}: C([0,1]) \rightarrow C([0,1])$ is essentially due to Galaz Fontes and Solís, [15].

Theorem 2.3. The Cesàro operator $\mathrm{C}: C([0,1]) \rightarrow C([0,1])$ is power bounded (hence, not hypercyclic) and mean ergodic but, not uniformly mean ergodic. 
Proof. Since $\|C\|_{o p}=1$ and $C^{n} \mathbf{1}=\mathbf{1}$ for each $n \in \mathbb{N}$, it follows that $\left\|C^{n}\right\|_{o p}=1$ for each $n \in \mathbb{N}$. Hence, $\mathrm{C}$ is power bounded; this also implies immediately that $\mathrm{C}$ cannot be hypercyclic. By [15, Theorem 3], for every $f \in C([0,1])$, the sequence $\left\{\mathrm{C}^{n} f\right\}_{n=1}^{\infty}$ converges to $f(0) \mathbf{1}$ in $C([0,1])$. This implies that the operator sequence of iterates $\left\{\mathrm{C}^{n}\right\}_{n=1}^{\infty}$ converges to the projection $P: C([0,1]) \rightarrow C([0,1])$ given by $f \mapsto P f:=f(0) \mathbf{1}$, in $\mathcal{L}_{s}(C([0,1]))$. Consequently, the arithmetic means $\left\{\mathrm{C}_{[n]}\right\}_{n=1}^{\infty}$ also converge to $P$ in $\mathcal{L}_{s}(C([0,1]))$, i.e., $\mathrm{C}$ is mean ergodic. Finally, suppose that C: $C([0,1]) \rightarrow C([0,1])$ is uniformly mean ergodic. By $[20, \S 2.2$, Theorem 2.7], the point 1 cannot be a limit point of the spectrum of $C$. This contradicts Proposition 2.1 .

It is of some interest to determine explicitly the closure $\overline{(I-\mathrm{C})(C([0,1]))}$ of the range $(I-\mathrm{C})(C([0,1]))$, and to give a necessary condition which ensures that $g \in C([0,1])$ belongs to $(I-\mathrm{C})(C([0,1]))$.

Proposition 2.4. (i) The closure $\overline{(I-\mathrm{C})(C([0,1]))}$ of the range $(I-\mathrm{C})(C([0,1]))$ of $(I-\mathrm{C})$ is precisely the space $Z:=\{f \in C([0,1]): f(0)=0\}$.

(ii) Let $g \in C([0,1])$ belong to $(I-\mathrm{C})(C([0,1]))$. Then $g(0)=0$ and, for each $x \in(0,1)$, the limit $\lim _{\varepsilon \rightarrow 0^{+}} \int_{\varepsilon}^{x} \frac{g(t)}{t} d t$ exists.

Proof. (i) Clearly, $Z$ is a closed subspace of $C([0,1])$. Since $C f(0)=f(0)$ for all $f \in C([0,1])$, the space $(I-\mathrm{C})(C([0,1])) \subseteq Z$. So, $\overline{(I-\mathrm{C})(C([0,1]))} \subseteq Z$.

For each $n \in \mathbb{N}$, direct calculation yields $(I-\mathrm{C}) x^{n}=\left(1-\frac{1}{n+1}\right) x^{n}$. It follows that

$$
\operatorname{span}\left\{x^{n}: n \in \mathbb{N}\right\} \subseteq(I-\mathrm{C})(C([0,1])) \subseteq Z .
$$

Fix $g \in Z$. By Weierstrass' theorem there exists a sequence of polynomials $\left\{P_{k}\right\}_{k=1}^{\infty}$ such that $P_{k} \rightarrow g$ uniformly on $[0,1]$. Since $\mathbf{1} \notin Z$, the polynomials $\left\{P_{k}\right\}_{k=1}^{\infty}$ may not lie in $(I-\mathrm{C})(C([0,1])) \subseteq Z$. However, it follows from $P_{k}(0) \rightarrow$ $g(0)=0$ that the sequence of polynomials $Q_{k}(x):=P_{k}(x)-P_{k}(0)$, for $x \in[0,1]$ and $k \in \mathbb{N}$, lies in the left-side of (2.2). Since also $Q_{k} \rightarrow g$ uniformly on $[0,1]$, we have (via (2.2)) that $g \in \overline{\operatorname{span}\left\{x^{n}: n \in \mathbb{N}\right\}}$ and the result is proved.

(ii) Let $f \in C([0,1])$ satisfy $(I-\mathrm{C}) f=g$. Then $g(0)=0$ and

$$
f(x)-\frac{1}{x} \int_{0}^{x} f(t) d t=g(x), \quad x \in(0,1] .
$$

The function $h:=(f-g) \in C([0,1])$ satisfies $h(x)=\frac{1}{x} \int_{0}^{x} f(t) d t$, for $x \in(0,1]$, and hence, $h$ is continuously differentiable on $(0,1]$. Since $x h(x)=\int_{0}^{x} f(t) d t$, for $x \in(0,1]$, we can conclude via differentiation that $h(x)+x h^{\prime}(x)=f(x)$, for $x \in(0,1]$. It follows that $h^{\prime}(x)=\frac{g(x)}{x}$ on $(0,1]$.

Fix $x \in(0,1]$. For each $\varepsilon \in(0, x)$, the continuity of $h^{\prime}$ on $[\varepsilon, x]$ implies that

$$
h(x)-h(\varepsilon)=\int_{\varepsilon}^{x} h^{\prime}(t) d t=\int_{\varepsilon}^{x} \frac{g(t)}{t} d t .
$$

As $h \in C([0,1])$, it follows that $\lim _{\varepsilon \rightarrow 0^{+}} \int_{\varepsilon}^{x} \frac{g(t)}{t} d t=h(x)-h(0)$ exists.

Remark 2.5. One can use Proposition 2.4 to provide an alternate, more transparent proof of the fact that $\mathrm{C}: C([0,1]) \rightarrow C([0,1])$ is not uniformly mean ergodic: Consider the continuous function $g(x):=-1 /(\log x)$, for $x \in(0,1 / 2]$, 
with $g(0):=0$ and $g(x):=1 /(\log 2)$, for $x \in[1 / 2,1]$. By Proposition 2.4(i), the function $g \in \overline{(I-\mathrm{C})(C([0,1]))}$. On the other hand, for every $\varepsilon \in(0,1 / 2)$, we have

$$
\int_{\varepsilon}^{1 / 2} \frac{g(t)}{t} d t=-\int_{\varepsilon}^{1 / 2} \frac{d t}{t \log t}=\log (-\log \varepsilon)-\log (\log 2),
$$

which tends to $\infty$ as $\varepsilon \rightarrow 0^{+}$. By Proposition 2.4(ii), it follows that $g \notin(I-$ C) $(C([0,1]))$, i.e., $(I-\mathrm{C})(C([0,1]))$ is not closed in $C([0,1])$. Then a result of M. Lin [24] yields that $C$ is not uniformly mean ergodic.

Theorem 2.6. The Cesàro operator $\mathrm{C}: C_{l}([0, \infty]) \rightarrow C_{l}([0, \infty])$ is power bounded (hence, not hypercyclic) and not mean ergodic. Moreover,

$$
\overline{(I-\mathrm{C})\left(C_{l}([0, \infty])\right)}=\left\{f \in C_{l}([0, \infty]): f(0)=f(\infty)=0\right\}
$$

Proof. For each $n \in \mathbb{N}$, we have $\left\|\mathrm{C}^{n}\right\|_{o p}=1$ and so $\mathrm{C}$ is power bounded. In particular, $\mathrm{C}$ is then not hypercyclic.

We first prove the identity (2.3). Clearly,

$$
Z:=\left\{f \in C_{l}([0, \infty]): f(0)=f(\infty)=0\right\}
$$

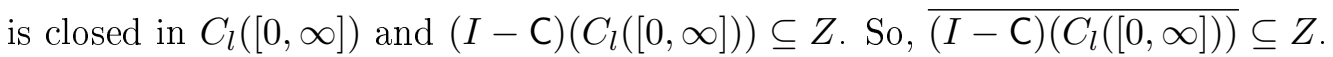

For each $m, n \in \mathbb{N}$, define $h_{m, n}(x)=x^{n}$ if $x \in[0, m]$ and $h_{m, n}(x)=m^{n}$ if $x \in$ $[m, \infty)$. Then $(I-\mathrm{C}) h_{m, n}(x)=\left(1-\frac{1}{n+1}\right) x^{n}$ if $x \in[0, m]$ and $(I-\mathrm{C}) h_{m, n}(x)=$ $\left(1-\frac{1}{n+1}\right) \frac{m^{n+1}}{x}$ if $x \in[m, \infty)$. For each $m, n \in \mathbb{N}$, let $g_{m, n}:=\frac{n+1}{n}(I-\mathrm{C}) h_{m, n}$. Then, for every $m, n \in \mathbb{N}$, we have $g_{m, n} \in(I-\mathrm{C})\left(C_{l}([0, \infty])\right)$ with $g_{m, n}(x)=x^{n}$ if $x \in[0, m]$ and $g_{m, n}(x)=\frac{m^{n+1}}{x}$ if $x \in[m, \infty)$. So, $\operatorname{span}\left\{g_{m, n}: m, n \in \mathbb{N}\right\} \subseteq$ $(I-\mathrm{C})\left(C_{l}([0, \infty])\right)$.

Fix $\psi \in Z$. For each $\varepsilon>0$, there is $M \in \mathbb{N}$ with $|\psi(x)| \leq \frac{\varepsilon}{3}$ whenever $x \geq M$ $($ as $\psi(\infty)=0)$. By Weierstrass' Theorem there is a polynomial $Q(x)=\sum_{j=1}^{r} a_{j} x^{j}$ with $Q(0)=0$ such that $|\psi(x)-Q(x)| \leq \frac{\varepsilon}{3}$ for $x \in[0, M]$. Observe that $|Q(M)| \leq$ $|Q(M)-\psi(M)|+|\psi(M)| \leq \frac{2 \varepsilon}{3}$. Moreover, the function $h:=\sum_{j=1}^{r} a_{j} g_{M, j}$ belongs to $(I-\mathrm{C})\left(C_{l}([0, \infty])\right)$ and coincides with $Q$ on $[0, M]$. Now, if $x \in[0, M]$, then

$$
|\psi(x)-h(x)|=|\psi(x)-Q(x)| \leq \frac{\varepsilon}{3}
$$

and, if $x \geq M$, then

$$
\begin{aligned}
& |\psi(x)-h(x)|=\left|\psi(x)-\sum_{j=1}^{r} a_{j} \frac{M^{j+1}}{x}\right| \leq|\psi(x)|+\frac{M}{x}\left|\sum_{j=1}^{r} a_{j} M^{j}\right| \\
& =|\psi(x)|+\frac{M}{x}|Q(M)| \leq \frac{\varepsilon}{3}+\frac{2 \varepsilon}{3}=\varepsilon .
\end{aligned}
$$

Accordingly, $\|\psi-h\|_{\infty} \leq \varepsilon$. It follows that $\psi \in \overline{(I-\mathrm{C})\left(C_{l}([0, \infty])\right)}$.

Finally, we show that the power bounded operator $C$ is not mean ergodic in $C_{l}([0, \infty])$. On the contrary, if $\mathrm{C}$ is mean ergodic, then $C_{l}([0, \infty])=\operatorname{Ker}(I-$ C) $\oplus \overline{(I-\mathrm{C})\left(C_{l}([0, \infty])\right)},[20, \S 2.1$, Theorem 1.3], and so the function $f(x)=$ $(\cos x) /(x+1) \in C_{l}([0, \infty])$ could be written as $f=c \mathbf{1}+g$ with $g(0)=g(\infty)=0$; see (2.3). This implies that $f(0)=c=f(\infty)$. But, $f(0)=1$ and $f(\infty)=0$ which gives a contradiction. 
Our next result for $C([0,1])$ is stated (correctly) in [23, Theorem 2.7]. However, the proof given there is incorrect as it is based on the claim that $\sigma_{p t}\left(\mathbf{C}^{\prime}\right)=\emptyset$, which is not the case. Indeed, the Dirac point measure $\delta_{0}$ induces the element of $(C([0,1]))^{\prime}$ given by $\delta_{0}: f \mapsto f(0)$, which satisfies $C^{\prime} \delta_{0}=\delta_{0}$. Hence, $\sigma_{p t}\left(\mathrm{C}^{\prime}\right) \neq \emptyset$. This result was also proved correctly in [16, Prop. 16].

Proposition 2.7. Neither of the two Cesàro operators $\mathrm{C}: C([0,1]) \rightarrow C([0,1])$ and $\mathrm{C}: C_{l}([0, \infty]) \rightarrow C_{l}([0, \infty])$ is supercyclic.

Proof. Proceeding by contradiction, suppose that there exists a supercyclic vector $g \in C([0,1])$ for C, i.e., the set $\left\{\lambda C^{n} g: \lambda \in \mathbb{C}, n \in \mathbb{N}_{0}\right\}$ is dense in $C([0,1])$. Then there exist a sequence $\left\{\lambda_{k}\right\}_{k=1}^{\infty} \subseteq \mathbb{C}$ and an increasing sequence $\left\{n_{k}\right\}_{k=1}^{\infty} \subseteq \mathbb{N}$ such that $\lambda_{k} \mathrm{C}^{n_{k}} g \rightarrow \mathbf{1}$ in $C([0,1])$ for $k \rightarrow \infty$. In particular, $\lambda_{k} \mathrm{C}^{n_{k}} g(0)=\lambda_{k} g(0) \rightarrow 1$ for $k \rightarrow \infty$ and so $g(0) \neq 0$. On the other hand, given any function $f \in C([0,1])$ such that $f \neq 0$ but $f(0)=0$ (eg., $f(x)=x$ for $x \in[0,1]$ ), there exist a sequence $\left\{\mu_{r}\right\}_{r=1}^{\infty} \subseteq \mathbb{C}$ and an increasing sequence $\left\{m_{r}\right\}_{r=1}^{\infty} \subseteq \mathbb{N}$ such that $\mu_{r} \mathrm{C}^{m_{r}} g \rightarrow f$ in $C([0,1])$ for $r \rightarrow \infty$. Hence, $\mu_{r} \mathrm{C}^{m_{r}} g(0)=\mu_{r} g(0) \rightarrow f(0)=0$ for $r \rightarrow \infty$ and so $\mu_{r} \rightarrow 0$ for $r \rightarrow \infty(\operatorname{as} g(0) \neq 0)$. Since $\left\|\mu_{r} C^{m_{r}} g\right\|_{\infty} \leq\left|\mu_{r}\right| \cdot\|g\|_{\infty}$ for all $r \in \mathbb{N}$, it follows that $\|f\|_{\infty}=0$; a contradiction to $f \neq 0$.

The proof for C: $C_{l}([0, \infty]) \rightarrow C_{l}([0, \infty])$ is similar. It suffices to replace the continuous function $f$ used there (i.e., $f(x)=x$, for $x \in[0,1])$ with $f \in C_{l}([0, \infty]$ ) given by $f(x)=x$ if $x \in[0,1]$ and $f(x)=1$ if $x \geq 1$.

\section{The Cesàro operator on the Fréchet space $C\left(\mathbb{R}^{+}\right)$}

The lc-topology of the Fréchet space $C\left(\mathbb{R}^{+}\right)$(see $\S 1$ ) is generated by the increasing sequence of seminorms

$$
q_{j}(f):=\max _{x \in[0, j]}|f(x)|, \quad f \in C\left(\mathbb{R}^{+}\right), j \in \mathbb{N} .
$$

For each $j \in \mathbb{N}$, we denote by $C([0, j])$ the Banach space of all $\mathbb{C}$-valued, continuous functions on $[0, j]$ endowed with the norm

$$
\|f\|_{j}:=\max _{x \in[0, j]}|f(x)|, \quad f \in C([0, j]) .
$$

For each $j \in \mathbb{N}$, let $Q_{j}: C\left(\mathbb{R}^{+}\right) \rightarrow C([0, j])$ and $Q_{j, j+1}: C([0, j+1]) \rightarrow$ $C([0, j])$ be the respective restriction maps, i.e., $Q_{j} f:=\left.f\right|_{[0, j]}$ for $f \in C\left(\mathbb{R}^{+}\right)$ and $Q_{j, j+1} f:=\left.f\right|_{[0, j]}$ for $f \in C([0, j+1])$. Clearly, $Q_{j, j+1} \circ Q_{j+1}=Q_{j}$ with $\left\|Q_{j} f\right\|_{j}=q_{j}(f)$ and $\left\|Q_{j, j+1} g\right\|_{j}=\|g\|_{j} \leq\|g\|_{j+1}$ for every $f \in C\left(\mathbb{R}^{+}\right)$, $g \in C([0, j+1])$ and $j \in \mathbb{N}$. Moreover, we have the projective limit $C\left(\mathbb{R}^{+}\right)=$ $\operatorname{proj}_{j \in \mathbb{N}}\left(C([0, j]), Q_{j, j+1}\right)$. Observe that all of the operators $Q_{j, j+1}$ and $Q_{j}$, for $j \in \mathbb{N}$, are surjective.

We investigate the Cesàro operator $C: C\left(\mathbb{R}^{+}\right) \rightarrow C\left(\mathbb{R}^{+}\right)$defined, for every $f \in C\left(\mathbb{R}^{+}\right)$, by $C f(0)=f(0)$ and $C f(x)=\frac{1}{x} \int_{0}^{x} f(t) d t$, for $x>0$. To do this we denote by $\mathrm{C}_{j}: C([0, j]) \rightarrow C([0, j])$ the Banach space operator defined by the same formulae but, now for $f \in C([0, j]), j \in \mathbb{N}$. It is routine to check that $\mathrm{C}_{j} Q_{j}=Q_{j} \mathrm{C}$ and $Q_{j, j+1} \mathrm{C}_{j+1}=\mathrm{C}_{j} Q_{j, j+1}$ for every $j \in \mathbb{N}$. The continuity of $\mathrm{C}$ on $C\left(\mathbb{R}^{+}\right)$is immediate from the inequalities $q_{j}(\mathrm{C} f) \leq q_{j}(f)$, for $f \in C\left(\mathbb{R}^{+}\right)$and $j \in \mathbb{N}$. For each $j \in \mathbb{N}$, define $T_{j}: C([0,1]) \rightarrow C([0, j])$ by $T_{j} g(x):=g(x / j)$, for $x \in[0, j]$ and $g \in C([0,1])$. The linear operator $T_{j}$ is an isometry with inverse given by $T_{j}^{-1} h(x):=h(j x)$, for $x \in[0,1]$ and $h \in C([0, j])$. Moreover, 
$T_{j} \mathrm{C}_{1}=\mathrm{C}_{j} T_{j}$, for each $j \in \mathbb{N}$. To see this, fix $f \in C([0,1])$ and $x \in[0, j]$. Since $\left(T_{j} \mathrm{C}_{1} f\right)(0)=\left(\mathrm{C}_{j} T_{j} f\right)(0)=f(0)$ we may assume that $x \in(0, j]$. Then

$$
\begin{aligned}
& \left(\mathrm{C}_{j} T_{j} f\right)(x)=\frac{1}{x} \int_{0}^{x}\left(T_{j} f\right)(t) d t=\frac{1}{x} \int_{0}^{x} f(t / j) d t \\
& =\frac{1}{x / j} \int_{0}^{x / j} f(s) d s=\left(\mathrm{C}_{1} f\right)(x / j)=\left(T_{j} \mathrm{C}_{1}\right) f(x) .
\end{aligned}
$$

It follows from $T_{j} \mathrm{C}_{1}=\mathrm{C}_{j} T_{j}$ that $T_{j} \mathrm{C}_{1}^{n}=\mathrm{C}_{j}^{n} T_{j}$ and hence, that $T_{j} \mathrm{C}_{1}^{n} T_{j}^{-1}=\mathrm{C}_{j}^{n}$ for all $j, n \in \mathbb{N}$. Since both $T_{j}, T_{j}^{-1}$ are isometries, we can conclude that $\left\|C_{j}^{n}\right\|_{o p}=$ $\left\|C_{1}^{n}\right\|_{o p}=1$, for each $j, n \in \mathbb{N}$, and that both

$$
\sigma\left(\mathrm{C}_{j} ; C([0, j])\right)=\sigma\left(\mathrm{C}_{1} ; C([0,1])\right)
$$

and

$$
\sigma_{p t}\left(\mathrm{C}_{j} ; C([0, j])\right)=\sigma_{p t}\left(\mathrm{C}_{1} ; C([0,1])\right),
$$

for each $j \in \mathbb{N}$. So, for each $j \in \mathbb{N}$, the operator $C_{j}$ is power bounded. Moreover, the identities $\left(\mathrm{C}_{j}\right)_{[n]}=T_{j}\left(\mathrm{C}_{1}\right)_{[n]} T_{j}^{-1}$, for $j, n \in \mathbb{N}$, together with Theorem 2.3 and Proposition 2.7 imply, for each $j \in \mathbb{N}$, that $C_{j}$ is mean ergodic but, not uniformly mean ergodic and not supercyclic (hence, not hypercyclic).

Theorem 3.1. The Cesàro operator $\mathrm{C}: C\left(\mathbb{R}^{+}\right) \rightarrow C\left(\mathbb{R}^{+}\right)$is power bounded and mean ergodic but, not uniformly mean ergodic and not supercyclic (hence, not hypercyclic). Moreover,

$$
\sigma\left(\mathrm{C} ; C\left(\mathbb{R}^{+}\right)\right)=\left\{\lambda \in \mathbb{C}:\left|\lambda-\frac{1}{2}\right| \leq \frac{1}{2}\right\}
$$

and

$$
\sigma_{p t}\left(\mathrm{C} ; C\left(\mathbb{R}^{+}\right)\right)=\sigma\left(\mathrm{C} ; C\left(\mathbb{R}^{+}\right)\right) \backslash\{0\} .
$$

Proof. All the assumptions of Lemmas 5.1 and 5.4 (in the Appendix) are satisfied with $X:=C\left(\mathbb{R}^{+}\right), X_{j}:=C([0, j]), S:=\mathrm{C} \in \mathcal{L}(X)$ and $S_{j}:=\mathrm{C}_{j} \in \mathcal{L}\left(X_{j}\right)$, for $j \in \mathbb{N}$.

By Theorem 2.3 the operator $\mathrm{C}_{1}: C([0,1]) \rightarrow C([0,1])$ is power bounded and mean ergodic. The comments prior to Theorem 3.1 ensure that $\mathrm{C}_{j}: C([0, j]) \rightarrow$ $C([0, j])$ is also power bounded and mean ergodic, for each $j \in \mathbb{N}$. So, Lemma 5.4 (i) \&(iii) in the Appendix yield that $\mathrm{C}: C\left(\mathbb{R}^{+}\right) \rightarrow C\left(\mathbb{R}^{+}\right)$is both power bounded and mean ergodic.

If $\mathrm{C}$ were uniformly mean ergodic on $C\left(\mathbb{R}^{+}\right)$, then also $\mathrm{C}_{1}: C([0,1]) \rightarrow C([0,1])$ would be uniformly mean ergodic by Lemma 5.4(ii) in the Appendix. This contradicts Theorem 2.3. So, C is not uniformly mean ergodic.

Observe that $\mathrm{C}_{1} Q_{1}=Q_{1} \mathrm{C}$ with $Q_{1}$ surjective. If $\mathrm{C}: C\left(\mathbb{R}^{+}\right) \rightarrow C\left(\mathbb{R}^{+}\right)$is supercyclic, then $\left\{\lambda C^{n} f: n \in \mathbb{N}_{0}, \lambda \in \mathbb{C}\right\}$ is dense in $C\left(\mathbb{R}^{+}\right)$for some $f \in C\left(\mathbb{R}^{+}\right)$. By the properties mentioned in the previous two sentences it follows, with $g:=$ $Q_{1} f \in C([0,1])$, that $\left\{\lambda C_{1}^{n} g: n \in \mathbb{N}_{0}, \lambda \in \mathbb{C}\right\}$ is dense in $C([0,1])$, i.e., $C_{1}$ is supercyclic in $C([0,1])$. This contradicts Proposition 2.7. So, $C$ is not supercyclic in $C\left(\mathbb{R}^{+}\right)$.

Concerning the spectra, by (5.2) of Lemma 5.1 (in the Appendix) and Proposition 2.1 we have via (3.3) that

$$
\sigma\left(\mathrm{C} ; C\left(\mathbb{R}^{+}\right)\right) \subseteq \cup_{j=1}^{\infty} \sigma\left(\mathrm{C}_{j} ; C([0, j])\right)=\sigma\left(\mathrm{C}_{1} ; C([0,1])\right) .
$$


On the other hand, for every $\lambda \in \sigma_{p t}\left(\mathrm{C}_{1} ; C([0,1])\right)=\left\{\lambda \in \mathbb{C}:\left|\lambda-\frac{1}{2}\right| \leq \frac{1}{2}\right\} \backslash\{0\}$ (see Proposition 2.1), the function $f_{\lambda}(x)=x^{\frac{1}{\lambda}-1}$, for $x \in[0,1]$, when defined by the same formula for all $x \in \mathbb{R}^{+}$, belongs to $C\left(\mathbb{R}^{+}\right)$and satisfies $C f_{\lambda}=\lambda f_{\lambda}$. So, $\lambda \in \sigma_{p t}\left(\mathrm{C} ; C\left(\mathbb{R}^{+}\right)\right)$and we have

$$
\sigma_{p t}\left(\mathrm{C}_{1} ; C([0,1])\right) \subseteq \sigma_{p t}\left(\mathrm{C} ; C\left(\mathbb{R}^{+}\right)\right) \subseteq \sigma\left(\mathrm{C} ; C\left(\mathbb{R}^{+}\right)\right) .
$$

Since the range $C\left(C\left(\mathbb{R}^{+}\right)\right) \subseteq C^{1}\left(\mathbb{R}^{+}\right)$with $C^{1}\left(\mathbb{R}^{+}\right)$a proper subspace of $C\left(\mathbb{R}^{+}\right)$, we see that $\mathrm{C}$ is not surjective and so $0 \in \sigma\left(\mathrm{C} ; C\left(\mathbb{R}^{+}\right)\right)$. So, we also have via Proposition 2.1 that

$$
\sigma_{p t}\left(\mathrm{C}_{1} ; C([0,1])\right) \cup\{0\}=\sigma\left(\mathrm{C}_{1} ; C([0,1])\right) \subseteq \sigma\left(\mathrm{C} ; C\left(\mathbb{R}^{+}\right)\right) .
$$

By (5.2) of Lemma 5.1 (in the Appendix) and (3.4), (3.6), it follows that

$$
\begin{aligned}
& \sigma\left(\mathrm{C}_{1} ; C([0,1])\right) \subseteq \sigma\left(\mathrm{C} ; C\left(\mathbb{R}^{+}\right)\right) \cup \cup_{j=1}^{\infty} \sigma_{p t}\left(\mathrm{C}_{j} ; C([0, j])\right) \\
& =\sigma\left(\mathrm{C} ; C\left(\mathbb{R}^{+}\right)\right) \cup \sigma_{p t}\left(\mathrm{C}_{1} ; C([0,1])\right) \subseteq \sigma\left(\mathrm{C} ; C\left(\mathbb{R}^{+}\right)\right) .
\end{aligned}
$$

Combined with (3.5) this yields that

$$
\sigma\left(\mathrm{C} ; C\left(\mathbb{R}^{+}\right)\right)=\sigma\left(\mathrm{C}_{1} ; C([0,1])\right)=\left\{\lambda \in \mathbb{C}:\left|\lambda-\frac{1}{2}\right| \leq \frac{1}{2}\right\} .
$$

Finally, by (5.3) of Lemma 5.1 (in the Appendix), we have

$$
\sigma_{p t}\left(\mathrm{C} ; C\left(\mathbb{R}^{+}\right)\right) \subseteq \cup_{j=1}^{\infty} \sigma_{p t}\left(\mathrm{C}_{j} ; C([0, j])\right)=\sigma_{p t}\left(\mathrm{C}_{1} ; C([0,1])\right) \subseteq \sigma_{p t}\left(\mathrm{C} ; C\left(\mathbb{R}^{+}\right)\right) .
$$

Thus, from Proposition 2.1 it follows that

$$
\sigma_{p t}\left(\mathrm{C} ; C\left(\mathbb{R}^{+}\right)\right)=\sigma_{p t}\left(\mathrm{C}_{1} ; C([0,1])\right)=\left\{\lambda \in \mathbb{C}:\left|\lambda-\frac{1}{2}\right| \leq \frac{1}{2}\right\} \backslash\{0\} .
$$

4. The Cesàro operator on the Fréchet space $L_{l o c}^{p}\left(\mathbb{R}^{+}\right), 1<p<\infty$

Recall that $L_{l o c}^{p}\left(\mathbb{R}^{+}\right), 1<p<\infty$, is the Fréchet space of all $\mathbb{C}$-valued, measurable functions $f$ on $\mathbb{R}^{+}$such that

$$
q_{j}(f):=\left(\int_{0}^{j}|f(x)|^{p} d x\right)^{1 / p}<\infty, \quad j \in \mathbb{N},
$$

endowed with the lc-topology generated by the increasing sequence of seminorms $\left\{q_{j}\right\}_{j \in \mathbb{N}}$.

Fix $1<p<\infty$. For each $j \in \mathbb{N}$, denote by $L^{p}([0, j])$ the Banach space of all $\mathbb{C}$ valued, measurable functions on $[0, j]$ with the norm $\|f\|_{j}:=\left(\int_{0}^{j}|f(x)|^{p} d x\right)^{1 / p}$, for $f \in L^{p}([0, j])$.

For each $j \in \mathbb{N}$, denote by $Q_{j}: L_{l o c}^{p}\left(\mathbb{R}^{+}\right) \rightarrow L^{p}([0, j])$ and $Q_{j, j+1}: L^{p}([0, j+$ 1]) $\rightarrow L^{p}([0, j])$ the respective restriction maps on $[0, j]$, i.e., $Q_{j} f:=\left.f\right|_{[0, j]}$ for $f \in$ $L_{l o c}^{p}\left(\mathbb{R}^{+}\right)$and $Q_{j, j+1} f:=\left.f\right|_{[0, j]}$ for $f \in L^{p}([0, j+1])$. Clearly, for each $j \in \mathbb{N}$, we have $Q_{j, j+1} \circ Q_{j+1}=Q_{j}$ with $\left\|Q_{j} f\right\|_{j}=q_{j}(f)$, for $f \in L_{l o c}^{p}\left(\mathbb{R}^{+}\right)$, and $\left\|Q_{j, j+1} g\right\|_{j}=$ $\|g\|_{j} \leq\|g\|_{j+1}$, for $g \in L^{p}([0, j+1])$. Observe that the maps $Q_{j}$ and $Q_{j, j+1}$ are surjective for all $j \in \mathbb{N}$. Moreover, $L_{l o c}^{p}\left(\mathbb{R}^{+}\right)=\operatorname{proj}_{j \in \mathbb{N}}\left(L^{p}([0, j]), Q_{j, j+1}\right)$.

We consider the Cesàro operator $C: L_{l o c}^{p}\left(\mathbb{R}^{+}\right) \rightarrow L_{l o c}^{p}\left(\mathbb{R}^{+}\right)$given by $C f(x):=$ $\frac{1}{x} \int_{0}^{x} f(t) d t$, for $x>0$ and all $f \in L_{l o c}^{p}\left(\mathbb{R}^{+}\right)$, which is well defined as $L^{p}([0, x]) \subseteq$ 
$L^{1}([0, x])$ for each $x>0$. For each $j \in \mathbb{N}$, denote by $C_{j}$ the operator defined in the same way on the Banach space $L^{p}([0, j])$. By Hardy's inequality, [19, p.240], the linear operators $\mathrm{C}_{\text {and }} \mathrm{C}_{j}, j \in \mathbb{N}$, are continuous. Moreover, it is routine to check that $\mathrm{C}_{j} Q_{j}=Q_{j} \mathrm{C}$ and $Q_{j, j+1} \mathrm{C}_{j+1}=\mathrm{C}_{j} Q_{j}$, for each $j \in \mathbb{N}$. More detailed information about the Fréchet space $L_{l o c}^{p}\left(\mathbb{R}^{+}\right)$can be found in [1], [2], [3], for example.

Fix $j \in \mathbb{N}$ and define $T_{j}: L^{p}([0,1]) \rightarrow L^{p}([0, j])$ by $\left(T_{j} f\right)(x):=f(x / j)$, for $x \in[0, j]$ and $f \in L^{p}([0,1])$. Then the linear operator $T_{j}$ is a bijection with norm $\left\|T_{j}\right\|_{o p}=j^{1 / p}$. Indeed, for every $f \in L^{p}([0,1])$, we have

$$
\|T f\|_{j}^{p}=\int_{0}^{j}|(T f)(x)|^{p} d x=\int_{0}^{j}|f(x / j)|^{p} d x=j \int_{0}^{1}|f(y)|^{p} d y=j\|f\|_{1}^{p} .
$$

The inverse of $T_{j}$ is the operator $T_{j}^{-1}: L^{p}([0, j]) \rightarrow L^{p}([0,1])$ given by $\left(T_{j}^{-1} f\right)(x):=$ $f(j x)$, for $x \in[0,1]$ and $f \in L^{p}([0, j])$, with $\left\|T_{j}^{-1}\right\|_{o p}=j^{-1 / p}$.

The same calculations as in $\S 3$ show that $\mathrm{C}_{j} T_{j}=T_{j} \mathrm{C}_{1}$, for $j \in \mathbb{N}$. It follows that $T_{j} \mathrm{C}_{1}^{n}=\mathrm{C}_{j}^{n} T_{j}$ and hence, also that $T_{j} \mathrm{C}_{1}^{n} T_{j}^{-1}=\mathrm{C}_{j}^{n}$ for all $j, n \in \mathbb{N}$. Accordingly, $\left\|C_{j}^{n}\right\|_{o p} \leq\left\|T_{j}\right\|_{o p}\left\|C_{1}^{n}\right\|_{o p}\left\|T_{j}^{-1}\right\|_{o p}=\left\|C_{1}^{n}\right\|_{o p}$. In a similar way it follows from $C_{1}^{n}=$ $T_{j}^{-1} C_{j}^{n} T_{j}$ that $\left\|C_{1}^{n}\right\|_{o p} \leq\left\|C_{j}^{n}\right\|_{o p}$ and hence, for every $j, n \in \mathbb{N}$, that $\left\|C_{j}^{n}\right\|_{o p}=$ $\left\|\mathrm{C}_{1}^{n}\right\|_{o p}=q^{n}$, where $\frac{1}{p}+\frac{1}{q}=1$.

We now collect some known results about the Cesàro operator $C: L^{p}([0,1]) \rightarrow$ $L^{p}([0,1]), 1<p<\infty$, that are needed below.

Theorem 4.1. The Cesàro operator $\mathrm{C}: L^{p}([0,1]) \rightarrow L^{p}([0,1]), 1<p<\infty$, is not power bounded and not mean ergodic. On the other hand, it is hypercyclic, chaotic and satisfies

$$
\sigma\left(\mathrm{C} ; L^{p}([0,1])\right)=\left\{\lambda \in \mathbb{C}:\left|\lambda-\frac{q}{2}\right| \leq \frac{q}{2}\right\}
$$

and

$$
\sigma_{p t}\left(\mathrm{C} ; L^{p}([0,1])\right)=\left\{\lambda \in \mathbb{C}:\left|\lambda-\frac{q}{2}\right|<\frac{q}{2}\right\} .
$$

The statements about the spectrum are due to G.M. Leibowitz, [21]; see also [22, Theorem 1]. León-Saavedra et al. have shown in [23, Theorems 2.3 and $2.6]$, that $\mathrm{C}$ is both hypercyclic and chaotic on $L^{p}([0,1])$ and so, it is not power bounded. Moreover, $\mathrm{C}$ cannot be mean ergodic because the spectrum of a mean ergodic operator is contained in the closed unit disc; see Section 1.

Theorem 4.2. Let $1<p<\infty$. The Cesàro operator $C: L_{l o c}^{p}\left(\mathbb{R}^{+}\right) \rightarrow L_{l o c}^{p}\left(\mathbb{R}^{+}\right)$ is not power bounded and not mean ergodic but, it is hypercyclic, chaotic and satisfies

and

$$
\sigma\left(\mathrm{C} ; L_{l o c}^{p}\left(\mathbb{R}^{+}\right)\right)=\left\{\lambda \in \mathbb{C}:\left|\lambda-\frac{q}{2}\right| \leq \frac{q}{2}\right\}
$$

$$
\sigma_{p t}\left(\mathrm{C} ; L_{l o c}^{p}\left(\mathbb{R}^{+}\right)\right)=\left\{\lambda \in \mathbb{C}:\left|\lambda-\frac{q}{2}\right|<\frac{q}{2}\right\} .
$$

Proof. All the assumptions of Lemmas 5.1 and 5.4 (in the Appendix) are satisfied with $X:=L_{l o c}^{p}\left(\mathbb{R}^{+}\right), X_{j}:=L^{p}([0, j]), S:=\mathrm{C} \in \mathcal{L}(X)$ and $S_{j}:=\mathrm{C}_{j} \in \mathcal{L}\left(X_{j}\right)$, for $j \in \mathbb{N}$. By Theorem 4.1 the operator $C_{1}: L^{p}([0,1]) \rightarrow L^{p}([0,1])$ is neither power bounded nor mean ergodic. So, by applying Lemma 5.1 (i)\&(iii) (in the Appendix) 
we can conclude that also C: $L_{l o c}^{p}\left(\mathbb{R}^{+}\right) \rightarrow L_{l o c}^{p}\left(\mathbb{R}^{+}\right)$is not power bounded and not mean ergodic.

Since $\left(C_{j}\right)_{[n]}=T_{j}\left(C_{1}\right)_{[n]} T_{j}^{-1}$, the operator $C_{j}$ is hypercyclic on $L^{p}([0, j])$ for all $j \in \mathbb{N}$ (cf. Theorem 4.1). On account of the comments immediately prior to Theorem 4.2 and the identities $\mathrm{C}_{j} Q_{j}=Q_{j} \mathrm{C}$, for each $j \in \mathbb{N}$, we can apply [10, Proposition 2.1] to conclude that $C$ is hypercyclic on $L_{l o c}^{p}\left(\mathbb{R}^{+}\right)$.

Theorem 4.1 and the identities $\mathrm{C}_{j}=T_{j} \mathrm{C}_{1} T_{j}^{-1}$, for each $j \in \mathbb{N}$, imply that

$$
\sigma\left(\mathrm{C}_{j} ; L^{p}([0, j])\right)=\sigma\left(\mathrm{C}_{1} ; L^{p}([0,1])\right)
$$

and

$$
\sigma_{p t}\left(\mathrm{C}_{j} ; L^{p}([0, j])\right)=\sigma_{p t}\left(\mathrm{C}_{1} ; L^{p}([0,1])\right)
$$

for each $j \in \mathbb{N}$. Accordingly, we can apply (5.2) of Lemma 5.1 to conclude, via (4.2), that

$$
\sigma\left(\mathrm{C} ; L_{l o c}^{p}\left(\mathbb{R}^{+}\right)\right) \subseteq \cup_{j=1}^{\infty} \sigma\left(\mathrm{C}_{j} ; L^{p}([0, j])\right)=\sigma\left(\mathrm{C}_{1} ; L^{p}([0,1])\right) .
$$

Now, if $\lambda \in \sigma_{p t}\left(C_{1} ; L^{p}([0,1])\right)=\left\{\lambda \in \mathbb{C}:\left|\lambda-\frac{q}{2}\right|<\frac{q}{2}\right\}$ (see Theorem 4.1), then $\operatorname{Re}\left(\frac{1}{\lambda}\right)>\frac{1}{q}$ and so the function $f_{\lambda}(x):=x^{\frac{1}{\lambda}-1}$ belongs to $L_{l o c}^{p}\left(\mathbb{R}^{+}\right)$and is an eigenvector of $\mathrm{C}$ corresponding to the eigenvalue $\lambda$. To see this, observe for every $j \in \mathbb{N}$ that

$$
\left(q_{j}\left(f_{\lambda}\right)\right)^{p}=\int_{0}^{j}\left|x^{\frac{1}{\lambda}-1}\right|^{p} d x=\int_{0}^{j} x^{p\left(\operatorname{Re}\left(\frac{1}{\lambda}\right)-1\right)} d x<\infty,
$$

as $p\left(\operatorname{Re}\left(\frac{1}{\lambda}\right)-1\right)>p\left(\frac{1}{q}-1\right)=-1$. Thus, $f_{\lambda} \in L_{l o c}^{p}\left(\mathbb{R}^{+}\right)$. It is routine to check that $\mathrm{C} f_{\lambda}=\lambda f_{\lambda}$. Hence,

$$
\sigma_{p t}\left(\mathrm{C}_{1} ; L^{p}([0,1])\right) \subseteq \sigma_{p t}\left(\mathrm{C} ; L_{l o c}^{p}\left(\mathbb{R}^{+}\right)\right) \subseteq \sigma\left(\mathrm{C} ; L_{l o c}^{p}\left(\mathbb{R}^{+}\right)\right) .
$$

So, by (5.2) of Lemma 5.1 it follows that

$$
\begin{aligned}
& \sigma\left(\mathrm{C}_{1} ; L^{p}([0,1])\right) \subseteq \sigma\left(\mathrm{C} ; L_{l o c}^{p}\left(\mathbb{R}^{+}\right)\right) \cup \cup_{j=1}^{\infty} \sigma_{p t}\left(\mathrm{C}_{j} ; L^{p}([0, j])\right) \\
& =\sigma\left(\mathrm{C} ; L_{l o c}^{p}\left(\mathbb{R}^{+}\right)\right) \cup \sigma_{p t}\left(\mathrm{C}_{1} ; L^{p}([0,1])\right) \subseteq \sigma\left(\mathrm{C} ; L_{l o c}^{p}\left(\mathbb{R}^{+}\right)\right) .
\end{aligned}
$$

Combined with (4.4) this shows that

$$
\sigma\left(\mathrm{C} ; L_{l o c}^{p}\left(\mathbb{R}^{+}\right)\right)=\sigma\left(\mathrm{C}_{1} ; L^{p}([0,1])\right)=\left\{\lambda \in \mathbb{C}:\left|\lambda-\frac{q}{2}\right| \leq \frac{q}{2}\right\} .
$$

Now, by (5.3) of Lemma 5.1 we obtain

$\sigma_{p t}\left(\mathrm{C} ; L_{l o c}^{p}\left(\mathbb{R}^{+}\right)\right) \subseteq \cup_{j=1}^{\infty} \sigma_{p t}\left(\mathrm{C}_{j} ; L^{p}([0, j])\right)=\sigma_{p t}\left(\mathrm{C}_{1} ; L^{p}([0,1])\right) \subseteq \sigma_{p t}\left(\mathrm{C} ; L_{l o c}^{p}\left(\mathbb{R}^{+}\right)\right)$, and hence, that

$$
\sigma_{p t}\left(\mathrm{C} ; L_{l o c}^{p}\left(\mathbb{R}^{+}\right)\right)=\sigma_{p t}\left(\mathrm{C}_{1} ; L^{p}([0,1])\right)=\left\{\lambda \in \mathbb{C}:\left|\lambda-\frac{q}{2}\right|<\frac{q}{2}\right\} .
$$

We already know that $\mathrm{C}$ is hypercyclic. It remains to show that $\mathrm{C}$ is chaotic in $L_{l o c}^{p}\left(\mathbb{R}^{+}\right)$. For this we need the following result.

Lemma 4.3. Let $1<p<\infty$ and the sequence $\left\{\alpha_{n}\right\}_{n=1}^{\infty} \subseteq \mathbb{C}$ satisfy $\operatorname{Re}\left(\alpha_{n}\right)>-\frac{1}{p}$ for each $n \in \mathbb{N}$. Suppose that $\left\{\alpha_{n}\right\}_{n=1}^{\infty}$ has an accumulation point in the open set $H_{p}^{+}=\left\{z \in \mathbb{C}: \operatorname{Re}(z)>-\frac{1}{p}\right\}$. Then

$$
Y:=\operatorname{span}\left\{x^{\alpha_{n}}: n \in \mathbb{N}\right\}
$$


is a dense subspace of $L_{l o c}^{p}\left(\mathbb{R}^{+}\right)$.

Proof. For each $\alpha \in H_{p}^{+}$, set $f_{\alpha}:=x^{\alpha}$, in which case $\operatorname{Re}(\alpha)>-\frac{1}{p}$ ensures that $f_{\alpha} \in L_{l o c}^{p}\left(\mathbb{R}^{+}\right)$. Suppose that $F \in\left(L_{l o c}^{p}\left(\mathbb{R}^{+}\right)\right)^{\prime}$ satisfies $F(g)=0$ for each $g \in Y$. Then $F\left(f_{\alpha_{n}}\right)=0$, for each $n \in \mathbb{N}$. By the structure of the dual space of $L_{l o c}^{p}\left(\mathbb{R}^{+}\right)$, [2], there exist $j \in \mathbb{N}$ and $h \in L^{q}\left(\mathbb{R}^{+}\right)$, where $\frac{1}{p}+\frac{1}{q}=1$, such that $\operatorname{supp}(h) \subseteq[0, j]$ and

$$
F(f)=\int_{0}^{j} f(x) h(x) d x, \quad f \in L_{l o c}^{p}\left(\mathbb{R}^{+}\right) .
$$

Define $\Phi: H_{p}^{+} \rightarrow \mathbb{C}$ by $\Phi(\alpha):=F\left(f_{\alpha}\right)=\int_{0}^{j} x^{\alpha} h(x) d x$, for $\alpha \in H_{p}^{+}$. The function $\Phi$ is analytic on $H_{p}^{+}$and vanishes on the sequence $\left\{\alpha_{n}\right\}_{n=1}^{\infty}$, which has an accumulation point in $H_{p}^{+}$. So, $\Phi$ is identically zero on $H_{p}^{+}$, i.e., $F\left(f_{\alpha}\right)=0$ for all $\alpha \in H_{p}^{+}$. In particular, $F$ vanishes on all $\mathbb{C}$-valued polynomials on $\mathbb{R}^{+}$. Since such polynomials form a dense subspace of $L_{l o c}^{p}\left(\mathbb{R}^{+}\right)$, it follows that $F=0$ on $L_{l o c}^{p}\left(\mathbb{R}^{+}\right)$. As $F$ is arbitrary, we can conclude via the Hahn-Banach theorem that $Y$ is dense in $L_{l o c}^{p}\left(\mathbb{R}^{+}\right)$.

Returning to showing that $\mathrm{C}$ is chaotic in $L_{l o c}^{p}\left(\mathbb{R}^{+}\right)$, it suffices to verify that the space

$$
H:=\operatorname{span}\left\{\operatorname{Ker}(\lambda I-\mathrm{C}): \lambda=e^{2 \pi i \theta} \text { for some } \theta \in \mathbb{Q}\right\}
$$

is dense in $L_{l o c}^{p}\left(\mathbb{R}^{+}\right)$; see [18, Proposition 2.33]. We already know that

$$
\sigma_{p t}\left(\mathrm{C} ; L_{l o c}^{p}\left(\mathbb{R}^{+}\right)\right)=\left\{\lambda \in \mathbb{C}:\left|\lambda-\frac{q}{2}\right|<\frac{q}{2}\right\} .
$$

Since $q>1$, we can select $\left\{\theta_{n}\right\}_{n=1}^{\infty} \subseteq \mathbb{Q}$ such that $\lambda_{n}:=e^{2 \pi i \theta_{n}} \in \sigma_{p t}\left(\mathrm{C} ; L_{\text {loc }}^{p}\left(\mathbb{R}^{+}\right)\right)$ for each $n \in \mathbb{N}$ with $\lambda_{n} \rightarrow 1$ as $n \rightarrow \infty$. Define $\beta_{n}:=\frac{1}{\lambda_{n}}-1$, for $n \in \mathbb{N}$. Since $\lim _{n \rightarrow \infty} \beta_{n}=0$, also $\lim _{n \rightarrow \infty} \operatorname{Re}\left(\beta_{n}\right)=0$ and so there exists $N \in \mathbb{N}$ such that $\operatorname{Re}\left(\beta_{n}\right)>-\frac{1}{p}$ for all $n>N$. Hence, the sequence $\alpha_{n}:=\beta_{n+N}$, for $n \in \mathbb{N}$, satisfies $\operatorname{Re}\left(\alpha_{n}\right)>-\frac{1}{p}$ for all $n \in \mathbb{N}$ and $\left\{\alpha_{n}\right\}_{n=1}^{\infty}$ has $0 \in H_{p}^{+}$as an accumulation point. Then, by Lemma 4.3 applied to $\left\{\alpha_{n}\right\}_{n=1}^{\infty}$, the space $Y:=\operatorname{span}\left\{x^{\alpha_{n}}: n \in \mathbb{N}\right\}$ is dense in $L_{l o c}^{p}\left(\mathbb{R}^{+}\right)$. Since $C x^{\alpha_{n}}=\lambda_{n} x^{\alpha_{n}}$ with $x^{\alpha_{n}} \in L_{l o c}^{p}\left(\mathbb{R}^{+}\right)$, for all $n \in \mathbb{N}$, it follows that $Y \subseteq H$. Hence, $\mathrm{C}$ has a dense set of periodic points, i.e., it is chaotic (being also hypercyclic).

\section{Appendix}

Here we collect a few relevant results concerning the spectrum and mean ergodic properties of continuous linear operators defined on certain classes of Fréchet spaces.

Lemma 5.1. Let $X$ be a Fréchet space and $S \in \mathcal{L}(X)$. Suppose that $X=$ $\operatorname{proj}_{j \in \mathbb{N}}\left(X_{j}, Q_{j, j+1}\right)$, with $X_{j}$ a Banach space (having norm \|\|$_{j}$ ) and linking maps $Q_{j, j+1} \in \mathcal{L}\left(X_{j+1}, X_{j}\right)$ which are surjective for all $j \in \mathbb{N}$, and suppose, for each $j \in \mathbb{N}$, that there exists $S_{j} \in \mathcal{L}\left(X_{j}\right)$ satisfying

$$
S_{j} Q_{j}=Q_{j} S
$$

where $Q_{j} \in \mathcal{L}\left(X, X_{j}\right), j \in \mathbb{N}$, denotes the canonical projection of $X$ onto $X_{j}$ (i.e., $Q_{j, j+1} \circ Q_{j+1}=Q_{j}$ ). Then

$$
\sigma(S) \subseteq \cup_{j=1}^{\infty} \sigma\left(S_{j}\right) \subseteq \sigma(S) \cup \cup_{j=1}^{\infty} \sigma_{p t}\left(S_{j}\right)
$$


Moreover,

$$
\sigma_{p t}(S) \subseteq \cup_{j=1}^{\infty} \sigma_{p t}\left(S_{j}\right) .
$$

Proof. It follows from (5.1) that

$$
\left(\lambda I_{j}-S_{j}\right) Q_{j}=Q_{j}(\lambda I-S)
$$

for all $j \in \mathbb{N}$ and $\lambda \in \mathbb{C}$, where $I_{j}$ denotes the identity map on $X_{j}$.

Fix any $\lambda \in \cap_{j=1}^{\infty} \rho\left(S_{j}\right)$. If $(\lambda I-S) x=0$ for some $x \in X$, then by (5.4) we have $\left(\lambda I_{j}-S_{j}\right) Q_{j} x=Q_{j}(\lambda I-S) x=0$ for all $j \in \mathbb{N}$. It follows that $Q_{j} x=0$ for all $j \in \mathbb{N}$. This implies that $x=0$ as $x \in X=\operatorname{proj}_{j \in \mathbb{N}}\left(X_{j}, Q_{j, j+1}\right)$. The proof of the surjectivity of $(\lambda I-S)$ follows as in the last part of the proof (cf. p.154) of Theorem 4.1 of [6] via (5.4) and the fact that $\left(\lambda I_{j}-S_{j}\right)$ is bijective for all $j \in \mathbb{N}$. As $X$ is a Fréchet space, we can conclude that $(\lambda I-S) \in \mathcal{L}(X)$ and so $\lambda \in \rho(S)$. This establishes that $\sigma(S) \subseteq \cup_{j=1}^{\infty} \sigma\left(S_{j}\right)$.

To verify the second containment in (5.2) we first observe that if $\mu \in \rho(S)$, then $(\mu I-S)$ is invertible in $\mathcal{L}(X)$ and hence, $\left(\mu I_{j}-S_{j}\right) \in \mathcal{L}\left(X_{j}\right)$ is surjective for all $j \in \mathbb{N}$; this follows routinely from (5.4) and the fact that each operator $Q_{j}$, for $j \in \mathbb{N}$, is surjective. Suppose that $\nu \in \rho(S) \backslash \cap_{j=1}^{\infty} \rho\left(S_{j}\right)$. Then $\nu \notin \rho\left(S_{j_{0}}\right)$ for some $j_{0} \in \mathbb{N}$, i.e., $\left(\nu I_{j_{0}}-S_{j_{0}}\right)$ is not invertible in $\mathcal{L}\left(X_{j_{0}}\right)$. Since $\left(\nu I_{j_{0}}-S_{j_{0}}\right)$ is surjective, it follows that $\nu \in \sigma_{p t}\left(S_{j_{0}}\right)$.

Now, let $\lambda \in \cup_{j=1}^{\infty} \sigma\left(S_{j}\right)$. If $\lambda \in \sigma(S)$, then there is nothing to prove. If $\lambda \notin \sigma(S)$, then $\lambda \in \rho(S)$. From the previous paragraph $\lambda \in \sigma_{p t}\left(S_{j_{0}}\right)$ for some $j_{0} \in \mathbb{N}$, i.e., $\lambda \in \cup_{j=1}^{\infty} \sigma\left(S_{j}\right)$. This establishes the second containment in (5.2). Thereby (5.2) has been proved.

To verify (5.3) let $\lambda \in\left(\cup_{j=1}^{\infty} \sigma_{p t}\left(S_{j}\right)\right)^{c}$, in which case $\left(\lambda_{j} I_{j}-S_{j}\right)$ is injective for each $j \in \mathbb{N}$. Suppose that $x \in X$ satisfies $(\lambda I-S) x=0$ in which case (5.4) implies that $\left(\lambda I_{j}-S_{j}\right) Q_{j} x=0$ for every $j \in \mathbb{N}$. Hence, $Q_{j} x=0$ for every $j \in \mathbb{N}$ and so $x=0$. This shows that $(\lambda I-S)$ is injective and so $\lambda \notin \sigma_{p t}(S)$, i.e., $\lambda \in\left(\sigma_{p t}(S)\right)^{c}$. Thereby (5.3) is established.

A Fréchet space $X$ is always a projective limit of continuous linear operators $R_{j}: X_{j+1} \rightarrow X_{j}$, for $j \in \mathbb{N}$, with each $X_{j}$ a Banach space. If $X_{j}$ and $R_{j}$ can be chosen such that each $R_{j}$ is surjective and $X$ is isomorphic to the projective limit $\operatorname{proj}_{j \in \mathbb{N}}\left(X_{j}, R_{j}\right)$, then $X$ is called a quojection, [9, Section 5]. Banach spaces and countable products of Banach spaces are quojections. In [27] Moscatelli gave the first examples of quojections which are not isomorphic to countable products of Banach spaces. Concrete examples of quojection Fréchet spaces are $\omega=\mathbb{C}^{\mathbb{N}}$, the spaces $L_{l o c}^{p}(\Omega)$, for $1 \leq p \leq \infty$, and $C^{(m)}(\Omega)$ for $m \in \mathbb{N}_{0}$, with $\Omega \subseteq \mathbb{R}^{N}$ any open set, all of which are isomorphic to countable products of Banach spaces. We refer the reader to the survey paper [26] for further information. Under the assumptions of Lemma 5.1 the Fréchet space $X$ there is necessarily a quojection. The same is true in Lemma 5.2 and Lemma 5.4 to follow.

Lemma 5.2. Let $X$ be a Fréchet space and $\left\{S_{n}\right\}_{n=1}^{\infty} \subseteq \mathcal{L}(X)$. Suppose that $X=\operatorname{proj}_{j \in \mathbb{N}}\left(X_{j}, Q_{j, j+1}\right)$, with $X_{j}$ a Banach space (having norm \|\|$_{j}$ ) and linking maps $Q_{j, j+1} \in \mathcal{L}\left(X_{j+1}, X_{j}\right)$ which are surjective for all $j \in \mathbb{N}$, and suppose, for each $j, n \in \mathbb{N}$, that there exists $S_{n}^{(j)} \in \mathcal{L}\left(X_{j}\right)$ satisfying

$$
S_{n}^{(j)} Q_{j}=Q_{j} S_{n}
$$


where $Q_{j} \in \mathcal{L}\left(X, X_{j}\right), j \in \mathbb{N}$, denotes the canonical projection of $X$ onto $X_{j}$ (i.e., $\left.Q_{j, j+1} \circ Q_{j+1}=Q_{j}\right)$. Then the following statements are equivalent.

(i) The limit $\tau_{b}$ - $\lim _{n \rightarrow \infty} S_{n}=: S$ exists in $\mathcal{L}_{b}(X)$.

(ii) For each $j \in \mathbb{N}$, the limit $\tau_{b}-\lim _{n \rightarrow \infty} S_{n}^{(j)}=: S^{(j)}$ exists in $\mathcal{L}_{b}\left(X_{j}\right)$.

In this case, the operators $S \in \mathcal{L}(X)$ and $S^{(j)} \in \mathcal{L}\left(X_{j}\right)$, for $j \in \mathbb{N}$, satisfy

$$
S x=\left(S^{(j)} x_{j}\right)_{j}, \quad x=\left(x_{j}\right)_{j} \in X .
$$

Proof. For each $j \in \mathbb{N}$, define $q_{j}(x):=\left\|Q_{j} x\right\|_{j}$ for $x \in X$. Then $\left\{q_{j}\right\}_{j=1}^{\infty} \subseteq \Gamma_{X}$ is a fundamental sequence generating the lc-topology of $X\left(\right.$ as $\left.X=\operatorname{proj}_{j \in \mathbb{N}}\left(X_{j}, Q_{j, j+1}\right)\right)$.

(i) $\Rightarrow\left(\right.$ ii). The existence in $\mathcal{L}_{b}(X)$ of the stated limit $S \in \mathcal{L}(X)$ ensures the existence (in the norm of $X_{j}$ ) of

$$
\lim _{n \rightarrow \infty} S_{n}^{(j)} Q_{j} x=\lim _{n \rightarrow \infty} Q_{j} S_{n} x=Q_{j} S x,
$$

for all $j \in \mathbb{N}$ and $x \in X$, via the continuity of $Q_{j}$ and (5.5). In fact, the weaker requirement that $S_{n} \rightarrow S$ in $\mathcal{L}_{s}(X)$ suffices for this.

Fix $j \in \mathbb{N}$. Define $S^{(j)}$ on $X_{j}=Q_{j}(X)$ by $S^{(j)}\left(Q_{j} x\right):=Q_{j} S x$, for $x \in X$. Then $S^{(j)} \in \mathcal{L}\left(X_{j}\right)$. Indeed, $S^{(j)}$ is well defined because if $Q_{j} x=Q_{j} x^{\prime}$ for some $x, x^{\prime} \in X$, then $Q_{j}\left(x-x^{\prime}\right)=0$ and so, via (5.5), $0=S_{n}^{(j)} Q_{j}\left(x-x^{\prime}\right)=Q_{j} S_{n}\left(x-x^{\prime}\right)$ for all $n \in \mathbb{N}$. Passing to the limit for $n \rightarrow \infty$, it follows that $0=Q_{j} S\left(x-x^{\prime}\right)$, i.e., $Q_{j} S x=Q_{j} S x^{\prime}$. Clearly, $S^{(j)}$ is linear as both $Q_{j}$ and $S$ are linear. Finally, since $S^{(j)} u=\lim _{n \rightarrow \infty} S_{n}^{(j)} u$, for each $u \in X_{j}$ (c.f. (5.7)) and $\left\{S_{n}^{(j)}\right\}_{n=1}^{\infty} \subseteq \mathcal{L}\left(X_{j}\right)$ with $X_{j}$ a Banach space, it follows from the Uniform Boundedness Principle that $S^{(j)}$ is continuous and hence, $S_{n}^{(j)} \rightarrow S^{(j)}$ in $\mathcal{L}_{s}\left(X_{j}\right)$ for $n \rightarrow \infty$. It is routine (via (5.5)) to check that $S^{(j)} Q_{j}=Q_{j} S$.

As noted above, $X$ is necessarily a quojection and so there exists $B \in \mathcal{B}(X)$ such that $\mathcal{U}_{j} \subseteq Q_{j}(B)$, [13, Proposition 1], where $\mathcal{U}_{j}$ is the closed unit ball of $X_{j}$. So, by (5.5) we have

$$
\begin{aligned}
& \sup _{u \in \mathcal{U}_{j}}\left\|\left(S_{n}^{(j)}-S^{(j)}\right) u\right\|_{j} \leq \sup _{x \in B}\left\|\left(S_{n}^{(j)}-S^{(j)}\right) Q_{j} x\right\|_{j} \\
& =\sup _{x \in B} \|\left(Q_{j}\left(S_{n}-S\right) x \|_{j}=\sup _{x \in B} q_{j}\left(\left(S_{n}-S\right) x\right)\right.
\end{aligned}
$$

for all $n \in \mathbb{N}$. Since $\sup _{x \in B} q_{j}\left(\left(S_{n}-S\right) x\right) \rightarrow 0$ for $n \rightarrow \infty$ (by assumption), it follows that $\sup _{u \in \mathcal{U}_{j}}\left\|\left(S_{n}^{(j)}-S^{(j)}\right) u\right\|_{j} \rightarrow 0$ for $n \rightarrow \infty$, i.e., $\tau_{b}$ - $\lim _{n \rightarrow \infty} S_{n}^{(j)}=S^{(j)}$. Since $j \in \mathbb{N}$ is arbitrary, the proof is complete.

(ii) $\Rightarrow\left(\right.$ i). Fix $x=\left(x_{j}\right)_{j} \in X=\operatorname{proj}_{j \in \mathbb{N}}\left(X_{j}, Q_{j, j+1}\right)$ and set $S x:=\left(S^{(j)} x_{j}\right)_{j}$. Then $S x \in X$. Indeed, $Q_{j} x=x_{j}$ for all $j \in \mathbb{N}$ and so, via (5.5), we have

$$
\begin{gathered}
Q_{j, j+1} S_{j+1} x_{j+1}=\lim _{n \rightarrow \infty} Q_{j, j+1} S_{n}^{(j+1)} Q_{j+1} x=\lim _{n \rightarrow \infty} Q_{j, j+1} Q_{j+1} S_{n} x \\
=\lim _{n \rightarrow \infty} Q_{j} S_{n} x=\lim _{n \rightarrow \infty} S_{n}^{(j)} Q_{j} x=S^{(j)} x_{j},
\end{gathered}
$$

for all $j \in \mathbb{N}$, i.e., $S x \in X$. Clearly, the linearity of the $S^{(j)}$ 's imply the linearity of the map $S: x \mapsto S x$, for $x \in X$. Moreover, the continuity of $S$ is a consequence of $X=\operatorname{proj}_{j \in \mathbb{N}}\left(X_{j}, Q_{j, j+1}\right)$. Next, fix $j \in \mathbb{N}$ and $B \in \mathcal{B}(X)$. Again via (5.5) we 
have

$$
\begin{gathered}
\sup _{x \in B} q_{j}\left(\left(S_{n}-S\right) x\right)=\sup _{x \in B} \|\left(Q_{j}\left(S_{n}-S\right) x\left\|_{j}=\sup _{x \in B}\right\|\left(S_{n}^{(j)}-S^{(j)}\right) Q_{j} x \|_{j}\right. \\
\quad=\sup _{u \in Q_{j}(B)}\left\|\left(S_{n}^{(j)}-S^{(j)}\right) u\right\|_{j}
\end{gathered}
$$

for all $n \in \mathbb{N}$. Since $Q_{j}(B) \in \mathcal{B}\left(X_{j}\right)$, it follows from the assumption (ii) that $\sup _{u \in Q_{j}(B)}\left\|\left(S_{n}^{(j)}-S^{(j)}\right) u\right\|_{j} \rightarrow 0$ for $n \rightarrow \infty$. Accordingly, for each $j \in \mathbb{N}$ and each $B \in \mathcal{B}(X)$ we have $\lim _{n \rightarrow \infty} \sup _{x \in B} q_{j}\left(\left(S_{n}-S\right) x\right)=0$, i.e., (i) holds.

Remark 5.3. A careful examination of the proof of Lemma 5.2 shows that the equivalence (i) $\Leftrightarrow$ (ii) remains valid if $\tau_{b}$ is replaced with $\tau_{s}$.

Lemma 5.4. Let $X=\operatorname{proj}_{j \in \mathbb{N}}\left(X_{j}, Q_{j . j+1}\right)$ be a Fréchet space and operators $S \in \mathcal{L}(X)$ and $S_{j} \in \mathcal{L}\left(X_{j}\right)$, for $j \in \mathbb{N}$, be given which satisfy the assumptions of Lemma 5.1 (with $Q_{j} \in \mathcal{L}\left(X, X_{j}\right), j \in \mathbb{N}$, denoting the canonical projection of $X$ onto $X_{j}$ and \|\|$_{j}$ being the norm in the Banach space $\left.X_{j}\right)$.

(i) $S \in \mathcal{L}(X)$ is power bounded if and only if each $S_{j} \in \mathcal{L}\left(X_{j}\right), j \in \mathbb{N}$, is power bounded.

(ii) $S \in \mathcal{L}(X)$ is uniformly mean ergodic if and only if each $S_{j} \in \mathcal{L}\left(X_{j}\right)$, $j \in \mathbb{N}$, is uniformly mean ergodic.

(iii) $S \in \mathcal{L}(X)$ is mean ergodic if and only if each $S_{j} \in \mathcal{L}\left(X_{j}\right), j \in \mathbb{N}$, is mean ergodic.

Proof. Let $\left\{q_{j}\right\}_{j=1}^{\infty} \subseteq \Gamma_{X}$ be the fundamental sequence of seminorms generating the lc-topology of $X$ as given in the proof of Lemma 5.2.

(i) Suppose that each $S_{j} \in \mathcal{L}\left(X_{j}\right), j \in \mathbb{N}$, is power bounded, i.e., there exists $M_{j}>0$ such that

$$
\left\|S_{j}^{n} u\right\|_{j} \leq M_{j}\|u\|_{j}, \quad u \in X_{j}, n \in \mathbb{N} .
$$

It follows from (5.1) that $S_{j}^{n} Q_{j}=Q_{j} S^{n}$ for all $j, n \in \mathbb{N}$. Fix $j \in \mathbb{N}$. Then, for each $n \in \mathbb{N}$ and $x \in X$ we have

$$
q_{j}\left(S^{n} x\right)=\left\|Q_{j} S^{n} x\right\|_{j}=\left\|S_{j}^{n} Q_{j} x\right\|_{j} \leq M_{j}\left\|Q_{j} x\right\|_{j}=M_{j} q_{j}(x) .
$$

Since $\left\{q_{j}\right\}_{j=1}^{\infty}$ generate the lc-topology of the Fréchet space $X$, it follows that $\left\{S^{n}\right\}_{n=1}^{\infty} \subseteq \mathcal{L}(X)$ is equicontinuous, i.e., $S$ is power bounded.

Conversely, suppose that $S$ is power bounded. Fix $j \in \mathbb{N}$ and let $\mathcal{U}_{j}$ be the closed unit ball of $X_{j}$. Since $X$ is a quojection, there exists $B \in \mathcal{B}(X)$ with $\mathcal{U}_{j} \subseteq$ $Q_{j}(B)$. Moreover, the power boundedness of $S$ implies that $C:=\cup_{n \in \mathbb{N}} S^{n}(B) \in$ $\mathcal{B}(X)$ and hence, there exists $M>0$ such that $q_{j}(z) \leq M$ for every $z \in C$. Let $u \in \mathcal{U}_{j}$. Then $u=Q_{j} x$ for some $x \in B$ and so

$$
\left\|S_{j}^{n} u\right\|_{j}=\left\|S_{j}^{n} Q_{j} x\right\|_{j}=\left\|Q_{j} S^{n} x\right\|_{j}=q_{j}\left(S^{n} x\right) \leq M,
$$

for every $n \in \mathbb{N}$. This implies that the operator norms satisfy $\left\|S_{j}^{n}\right\|_{o p} \leq M$, for $n \in \mathbb{N}$. Accordingly, $S_{j} \in \mathcal{L}\left(X_{j}\right)$ is power bounded.

(ii) For each $n \in \mathbb{N}$ define $\tilde{S}_{n}:=S_{[n]} \in \mathcal{L}(X)$ and $\tilde{S}_{n}^{(j)}:=\left(S_{j}\right)_{[n]} \in \mathcal{L}\left(X_{j}\right)$, for $j \in \mathbb{N}$. It follows from (5.1) that $\tilde{S}_{n}^{(j)} Q_{j}=Q_{j} \tilde{S}_{n}$, for $j, n \in \mathbb{N}$. Accordingly, we can apply Lemma 5.2 (with $\tilde{S}_{n}$ in place of $S_{n}$ and $\tilde{S}_{n}^{(j)}$ in place of $S_{n}^{(j)}$ ) to conclude 
that $S$ is uniformly mean ergodic if and only if each $S_{j}, j \in \mathbb{N}$, is uniformly mean ergodic.

(iii) Apply the same argument as in part (ii) but now apply Lemma 5.2 with $\tau_{s}$ in place of $\tau_{b}$; see Remark 5.3.

Acknowledgements. The research of the first two authors was partially supported by the projects MTM2010-15200 and GVA Prometeo II/2013/013 (Spain). The second author gratefully acknowledges the support of the Alexander von Humboldt Foundation.

\section{REFERENCES}

[1] A.A. Albanese, Primary products of Banach spaces. Arch. Math. 66 (1996), 397-405.

[2] A.A. Albanese, On subspaces of the spaces $L_{\mathrm{loc}}^{p}$ and of their strong duals. Math. Nachr. 197 (1999), 5-18.

[3] A.A. Albanese, V.B. Moscatelli, Complemented subspaces of sums and products of copies of $L^{1}[0,1]$. Rev. Mat. Univ. Complut. Madrid 9 (1996), 275-287.

[4] A.A. Albanese, J. Bonet, W.J. Ricker, Mean ergodic operators in Fréchet spaces. Ann. Acad. Sci. Fenn. Math. 34 (2009), 401-436.

[5] A.A. Albanese, J. Bonet, W.J. Ricker, On mean ergodic operators. In: Vector Measures, Integration and Related Topics, G.P. Curbera et. al. (Eds), Operator Theory: Advances and Applications 201, Birkhäuser Verlag, Basel, 2010, pp. 1-20.

[6] A.A. Albanese, J. Bonet, W.J. Ricker, $C_{0}$-semigroups and mean ergodic operators in a class of Fréchet spaces. J. Math. Anal. Appl. 365 (2010), 142-157.

[7] A.A. Albanese, J. Bonet, W.J. Ricker, Convergence of arithmetic means of operators in Fréchet spaces. J. Math. Anal. Appl. 401 (2013), 160-173.

[8] F. Bayart, E. Matheron, Dynamics of Linear Operators. Cambridge Tracts in Mathematics, 179. Cambridge University Press, Cambridge, 2009.

[9] S.F. Bellenot, E. Dubinsky, Fréchet spaces with nuclear Köthe quotients. Trans. Amer. Math. Soc. 273 (1982), 579-594.

[10] J. Bonet, L. Frerick, A. Peris, J. Wengenroth, Transitive and hypercyclic operators on locally convex spaces. Bull. London Math. Soc. 37 (2005), 254-264.

[11] D.W. Boyd, The spectrum of the Cesàro operator. Acta Sci. Math. (Szeged) 29 (1968), $31-34$.

[12] A. Brown, P.R. Halmos, A.L. Shields, Cesàro operators. Acta Sci. Math. (Szeged) 26 (1965), $125-137$.

[13] S. Dierolf, D.N. Zarnadze, A note on strictly regular Fréchet spaces. Arch. Math. 42 (1984), $549-556$

[14] N. Dunford, J.T. Schwartz, Linear Operators I: General Theory (2nd printing), WileyInterscience, New York, 1964.

[15] F. Galaz Fontes, F. J. Solís, Iterating the Cesàro operators. Proc. Amer. Math. Soc. 136 (2008), 2147-2153.

[16] F. Galaz Fontes, R. W. Ruiz-Aguilar, Grados de ciclicidad de los operadores de CesàroHardy, Misc. Mat. 57 (2013), 103-117.

[17] M. González, F. León-Saavedra, Cyclic behaviour of the Cesàro operator on $L_{2}(0,+\infty)$. Proc. Amer. Math. Soc. 137 (2009), 2049-2055.

[18] Karl-G. Grosse-Erdmann, A. Peris Manguillot, Linear Chaos. Universitext, Springer Verlag, London 2011.

[19] G.H. Hardy, J.E. Littlewood, G. Pólya, Inequalities. Reprint of the 1952 edition. Cambridge Mathematical Library. Cambridge University Press, Cambridge, 1988.

[20] U. Krengel, Ergodic Theorems. de Gruyter Studies in Mathematics, 6. Walter de Gruyter Co., Berlin, 1985.

[21] G.M. Leibowitz, Spectra of finite range Cesàro operators. Acta Sci. Math. (Szeged) 35 (1973), 27-28. 
[22] G.M. Leibowitz, The Cesàro operators and their generalizations: examples in infinitedimensional linear analysis. Amer. Math. Monthly 80 (1973), 654-661.

[23] F. León-Saavedra, A. Piqueras-Lerena, J.B. Seoane-Sepúlveda, Orbits of Cesàro type operators. Math. Nachr. 282 (2009), 764-773.

[24] M. Lin, On the uniform ergodic theorem. Proc. Amer. Math. Soc. 43 (1974), 337-340.

[25] R. Meise, D. Vogt, Introduction to Functional Analysis. Oxford Graduate Texts in Mathematics, 2. The Clarendon Press. Oxford University Press, New York, 1997.

[26] G. Metafune, V.B. Moscatelli, Quojections and prequojections. in "Advances in the Theory of Fréchet spaces", T. Terzioğlu (Ed.), NATO ASI Series, 287, Kluwer Academic Publishers, Dordrecht, 1989, pp. 235-254.

[27] V.B. Moscatelli, Fréchet spaces without norms and without bases. Bull. London Math. Soc. 12 (1980), 63-66.

[28] K. Piszczek, Quasi-reflexive Fréchet spaces and mean ergodicity. J. Math. Anal. Appl. 361 (2010), 224-233.

[29] K. Piszczek, Barrelled spaces and mean ergodicity. Rev. R. Acad. Cienc. Exactas Fis. Nat. Ser. A. Math. RACSAM 104 (2010), 5-11.

[30] K. Yosida, Functional Analysis. Sixth Edition, Springer-Verlag, Berlin, 1980.

Angela A. Albanese, Dipartimento di Matematica e Fisica "E. De Giorgi", Università del Salento- C.P.193, I-73100 Lecce, Italy

E-mail address: angela.albanese@unisalento.it

José Bonet, Instituto Universitario de Matemática Pura y Aplicada iUmpa, Universidad Politécnica de Valencia, E-46071 Valencia, Spain

E-mail address: jbonet@mat.upv.es

Werner J. Ricker, Math.-Geogr. Fakultät, Katholische Universität EichstättIngolstadt, D-85072 Eichstätt, Germany

E-mail address: werner.ricker@ku-eichstaett.de 